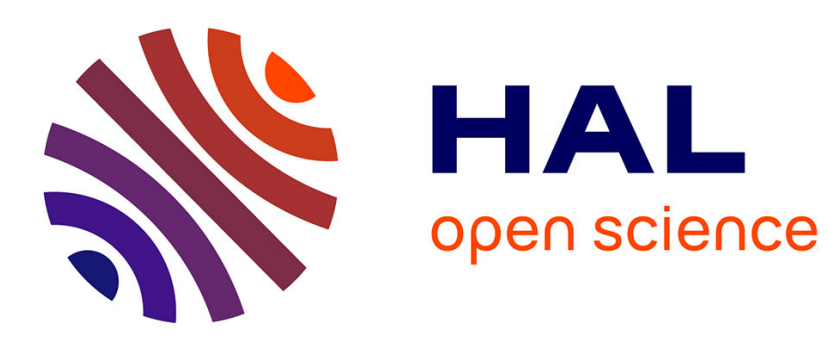

\title{
A polynomial-time algorithm for computing shortest paths of bounded curvature amidst moderate obstacles
}

Jean-Daniel Boissonnat, Sylvain Lazard

\section{To cite this version:}

Jean-Daniel Boissonnat, Sylvain Lazard. A polynomial-time algorithm for computing shortest paths of bounded curvature amidst moderate obstacles. International Journal of Computational Geometry and Applications, 2003, 13 (3), pp.189-229. 10.1142/S0218195903001128 . inria-00099509

\section{HAL Id: inria-00099509 \\ https://hal.inria.fr/inria-00099509}

Submitted on 15 Dec 2009

HAL is a multi-disciplinary open access archive for the deposit and dissemination of scientific research documents, whether they are published or not. The documents may come from teaching and research institutions in France or abroad, or from public or private research centers.
L'archive ouverte pluridisciplinaire HAL, est destinée au dépôt et à la diffusion de documents scientifiques de niveau recherche, publiés ou non, émanant des établissements d'enseignement et de recherche français ou étrangers, des laboratoires publics ou privés. 


\title{
A POLYNOMIAL-TIME ALGORITHM FOR COMPUTING SHORTEST PATHS OF BOUNDED CURVATURE AMIDST MODERATE OBSTACLES*
}

\author{
JEAN-DANIEL BOISSONNAT \\ INRIA Sophia-Antipolis, BP 93, \\ 06902 Sophia Antipolis Cedex, France. \\ Jean-Daniel.Boissonnat@inria.fr \\ SYLVAIN LAZARD \\ INRIA Lorraine - LORIA, 615 rue du jardin botanique, B.P. 101, \\ 54602 Villers-les-Nancy Cedex, France. \\ Sylvain.Lazard@inria.fr \\ Received (received date) \\ Revised (revised date) \\ Communicated by (Name)
}

\begin{abstract}
In this paper, we consider the problem of computing shortest paths of bounded curvature amidst obstacles in the plane. More precisely, given two prescribed initial and final configurations (specifying the location and the direction of travel) and a set of obstacles in the plane, we want to compute a shortest $C^{1}$ path joining those two configurations, avoiding the obstacles, and with the further constraint that, on each $C^{2}$ piece, the radius of curvature is at least 1 . In this paper, we consider the case of moderate obstacles and present a polynomial-time exact algorithm to solve this problem.
\end{abstract}

Keywords: Non-holonomic motion planning; curvature-constrained shortest paths; mobile robot; computational geometry.

\section{Introduction}

In this paper, we consider the problem of computing shortest paths of bounded curvature amidst obstacles in the plane, SBC path for short. More precisely, given two prescribed initial and final configurations (specifying the location and the direction of travel) and a set of obstacles in the plane, we want to compute a shortest $C^{1}$ path joining those two configurations, avoiding the obstacles, and with the further constraint that the radius of curvature is at least 1 on each $C^{2}$ piece. This question appears in many applications and goes back to Markov who studied the problem for joining pieces of railways. More recently, a great deal of attention has been paid

*A preliminary version of this paper appeared in Ref. 7. This work was done while the second author was a PhD student at INRIA Sophia-Antipolis. 
to this question in the context of non-holonomic robot motion planning. ${ }^{2,17,20,19} \mathrm{~A}$ robot is said to be non-holonomic if some kinematics constraints locally restricts the authorized directions for its velocity. A typical non-holonomic robot is a car: assuming no slipping of the wheels on the ground, the velocity of the midpoint between the two rear wheels of the car is always tangent to the car axis. Though the problem considered in this paper is one of the simplest instances of non-holonomic motion planning, it is still far from being well understood.

Even in the absence of obstacles, the problem is not easy. Dubins ${ }^{12}$ proved that any $\mathrm{SBC}$ path takes one of the following forms $C S C$ or $C C C$, where $C$ means a circular arc of radius 1 and $S$ a straight line segment. The proof in Dubins' paper is quite long and intricate. Recently, a much simpler proof has been obtained ${ }^{5,22}$ using the Minimum Principle of Pontryagin (a central result in Control Theory) and a complete characterization of SBC paths has also been established ${ }^{8}$.

The problem becomes much harder in the presence of obstacles. First, notice that it follows from Dubins' paper (see Refs. 12 or 14, 15) or from a theorem in Control Theory (see Refs. 9 Theorem 9.2.i or 18) that there exists a SBC path amidst obstacles and joining two given configurations as soon as there exists a $\mathrm{BC}$ path, i.e., a (not necessarily optimal) $C^{1}$ path joining the two given configurations, avoiding the obstacles and where the radius of curvature is everywhere (where it is defined) greater or equal to 1. Moreover, a SBC path is a concatenation of subpaths either contained in the boundary of some obstacles or connecting two obstacle boundaries (considering the initial and final configurations as point obstacles); each subpath joining two obstacle boundaries is a Dubins' path, i.e., a path of type $C S C$ or $C C C$. Computing a shortest path seems however a formidable task. Even if we remove the requirement for the path to be a shortest one and look for a $\mathrm{BC}$ path (instead of a SBC path), no polynomial-time algorithm is known. In Ref. 13, Fortune and Wilfong present an exact algorithm that can decide if a BC path exists but does not generate such a path if one exists. This algorithm runs in time and space that is exponential with respect to the number $n$ of corners of the environment and the number of bits used to specify the positions of the corners. By the remark above, this algorithm can also decide if a SBC path exists.

For computing SBC paths, only approximate algorithms have been proposed in the literature. Jacobs and Canny discretize the problem and calculate a path that approximates the shortest one in time $O\left(n^{2}\left(\frac{n+L}{\varepsilon}\right) \log n+\frac{(n+L)^{2}}{\varepsilon^{2}}\right)$, where $\varepsilon$ describes the closeness of the approximation and $L$ is the total edge length of the obstacle boundaries. ${ }^{15}$ Very recently, Wang and Agarwal improved on this result and proposed an algorithm whose time complexity is $O\left(\frac{n^{2}}{\varepsilon^{2}} \log n\right)$, and thus does not depend on $L .{ }^{23}$ In another recent paper ${ }^{1}$, Agarwal, Raghavan and Tamaki have considered a restricted class of obstacles, the so-called moderate obstacles: an obstacle is said to be moderate if it is convex and if its boundary is a differentiable curve whose radius of curvature is everywhere greater or equal to 1 . This restriction is quite strong but valid in many practical situations. Under the assumption that all 
the obstacles are disjoint and moderate, Agarwal et al. show that an approximate SBC path can be computed in $O\left(n^{2} \log n+1 / \varepsilon\right)$ time.

In this paper, we consider also the case of moderate obstacles (in a more restrictive sense than Agarwal et al.) and present a polynomial-time algorithm to compute a SBC path (assuming that the roots of some polynomials of bounded degree can be computed in constant time). To the best of our knowledge, this is the first polynomial-time exact algorithm for a nontrivial instance of the problem.

The paper is organized as follows. In Section 2, we introduce some notations and show that the problem reduces to finding Euclidean shortest paths when the initial and final positions are sufficiently far apart and sufficiently far from the obstacles. In Sections 3 and 4, we characterize the circular arcs that can appear in a SBC path. In Section 5, we study a particular class of subpaths. Finally, we describe in Section 6 an algorithm for computing shortest paths of bounded curvature between given configurations in the presence of moderate obstacle.

\section{Preliminaries}

First, we give some definitions and notations. Let $\Omega$ be a set of open obstacles. In this paper, the obstacles are assumed to be disjoint and moderate. An obstacle is said to be moderate if it is convex and if its boundary is a differentiable curve made of line segments and circular arcs of unit radius. For convenience and without a real loss of generality, we assume that no two edges of the obstacles are parallel. A path that avoids the obstacles is called free. In the sequel, a free SBC path is simply called an optimal path.

Let $\omega_{S}=\left(S, \vec{U}_{S}\right)$ and $\omega_{T}=\left(T, \vec{U}_{T}\right)$ be two configurations where $S$ and $T$ specify the location and $\vec{U}_{S}$ and $\vec{U}_{T}$ specify the direction of travel. Let $\mathcal{P}$ denote an optimal path joining $\omega_{S}$ to $\omega_{T}$.

As mentioned in the introduction, an optimal path $\mathcal{P}$ is a concatenation of circular arcs of unit radius, straight line segments and arcs contained in the boundary of some obstacles. A maximal circular arc of $\mathcal{P}$ that coincides with the boundary of an obstacle is called an $O$-segment. A $C$-segment is a maximal circular $\operatorname{arc}$ of $\mathcal{P}$ that does not coincide with the boundary of an obstacle. An $S$-segment is a maximal line segment of $\mathcal{P}$; an $S$-segment may coincide (partially or entirely) with the boundary of an obstacle. An optimal path is thus a concatenation of $O, C$ and $S$-segments. With these definitions, we ensure that two consecutive $O, C$ or $S$-segments in $\mathcal{P}$ cannot be supported by the same line or circle. We assume that the length of the $O, C$ and $S$-segments is never zero unless specified otherwise. In the following, we associate to a path the sequence of types $(O, C$ or $S)$ of its segments.

The first and last segments are called terminal. A terminal segment is, in general, a $C$-segment; we denote it by $C_{t}$. A $C$-segment (or a circle of unit radius) is denoted by $\bar{C}$ if it is tangent to at least one obstacle. A $C$-segment (or a circle of unit radius) is called anchored and denoted by $\overline{\bar{C}}$ if it is either tangent to at least two obstacles, or tangent to one obstacle and adjacent to a terminal $C$-segment, or if it is terminal. 
Our first theorem states that, when the initial and final positions are sufficiently far apart and sufficiently far from the obstacles, the optimal path is an Euclidean shortest path for an augmented set of obstacles.

Let $M$ be a point of an optimal path $\mathcal{P}$ and let $C_{L}(M)\left(\operatorname{resp} . C_{R}(M)\right)$ be the unit circle tangent to $\mathcal{P}$ at $M$ and lying on the left (resp. right) side of path $\mathcal{P}$ oriented from $S$ to $T . C_{L}(M)$ is oriented counterclockwise and $C_{R}(M)$ is oriented clockwise. An arc of one of these circles will be oriented accordingly.

Let $C_{S}^{\prime}$ (resp. $C_{T}^{\prime}$ ) be the circle tangent to $C_{L}(S)$ and $C_{R}(S)\left(\operatorname{resp} . C_{L}(T)\right.$ and $C_{R}(T)$ ) that does not intersect the ray $\left(S, \vec{U}_{S}\right)$ (resp. the ray $\left(T,-\vec{U}_{T}\right)$ ) (see Figure 1). Let $\mathcal{R}_{S}$ (resp. $\mathcal{R}_{T}$ ) be the open shaded region limited by $C_{L}(S), C_{R}(S)$ and $C_{S}^{\prime}$ (resp. $C_{L}(T), C_{R}(T)$ and $\left.C_{T}^{\prime}\right)$ as shown in Figure 1.
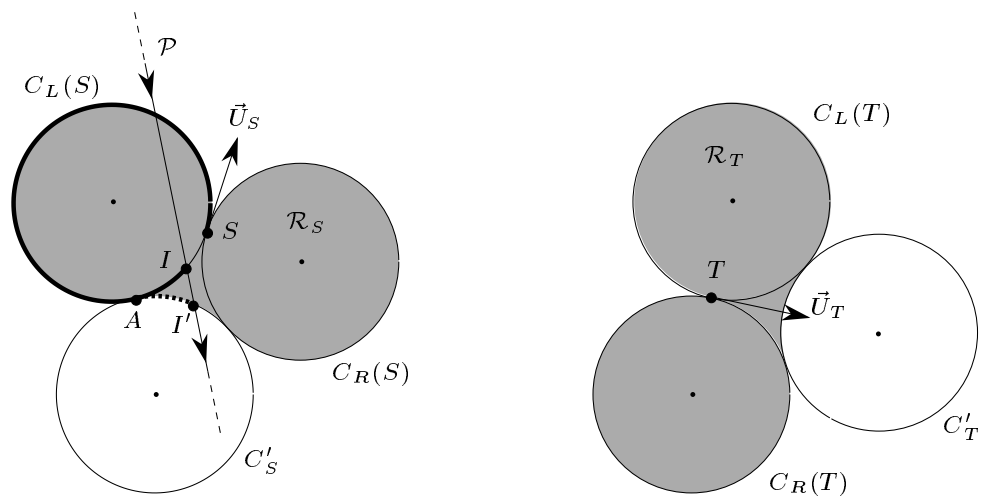

Fig. 1. Regions $\mathcal{R}_{S}$ and $\mathcal{R}_{T}$.

Lemma 1. If $\mathcal{R}_{S}$ and $\mathcal{R}_{T}$ are disjoint and do not intersect the obstacles, then $\mathcal{P}$ does not intersect $\mathcal{R}_{S} \cup \mathcal{R}_{T}$.

Proof. We assume for a contradiction that $\mathcal{P}$ intersects $\mathcal{R}_{S}$. We consider first the case where $\mathcal{P}$ does not intersect $\mathcal{R}_{T}$. Since $\mathcal{P}$ is a path of bounded curvature, $\mathcal{P}$ intersects $C_{L}(S)$ or $C_{R}(S)$. We assume for simplicity that $\mathcal{P}$ is never tangential to $C_{L}(S)$ or $C_{R}(S)$ except possibly at $S$. Let $I$ be the last intersection point (along $\mathcal{P}$ ) between $\mathcal{P}$ and $C_{L}(S) \cup C_{R}(S)$; assume, without loss of generality, that $I \in C_{L}(S)$. Let $I^{\prime}$ be the last intersection point (along $\mathcal{P}$ ) between $\mathcal{P}$ and $\mathcal{R}_{S}$ and let $I I^{\prime}$ be the part of $\mathcal{P}$ from $I$ to $I^{\prime}$ (notice that $I$ and $I^{\prime}$ can coincide). We denote by $A$ the point common to $C_{L}(S)$ and $C_{S}^{\prime}$ (see Figure 1 ).

First, we assume that $I \neq S$. Let $S I$ be the arc of $C_{L}(S)$, oriented as $C_{L}(S)$, starting at $S$ and ending at $I$. Let $\mathcal{P}^{\prime}$ be the concatenation of $S I$ and the part of $\mathcal{P}$ from $I$ to $T . \mathcal{P}^{\prime}$ is not a path of bounded curvature but it is shorter than $\mathcal{P}$ since the shortest path of bounded curvature from $\omega_{S}$ to $I$ (the orientation at $I$ 
is not specified) is the $\operatorname{arc} S I .{ }^{4}$ Let $\mathcal{P}^{\prime \prime}$ be the path obtained by modifying $\mathcal{P}^{\prime}$ as follows: if $I^{\prime}=I$ then $\mathcal{P}^{\prime \prime}=\mathcal{P}^{\prime}$, otherwise, we replace the $\operatorname{arc} A I$ of $C_{L}(S)$ and $I I^{\prime}$ by the short circular $\operatorname{arc} A I^{\prime}$ of $C_{S}^{\prime}$. Path $\mathcal{P}^{\prime \prime}$ is shorter than $\mathcal{P}^{\prime}$, thus $\mathcal{P}^{\prime \prime}$ is shorter than $\mathcal{P}$. Moreover, $\mathcal{P}^{\prime \prime}$ avoids $\mathcal{R}_{S}$ by construction, and avoids also all the moderate obstacles and $\mathcal{R}_{T}$ by hypothesis. Hence, the Euclidean shortest $\mathcal{P}^{\prime \prime \prime}$ path from $S$ to $T$ avoiding $\Omega, \mathcal{R}_{S}$ and $\mathcal{R}_{T}$ is shorter than $\mathcal{P}$. That yields a contradiction because this Euclidean shortest path is a path of bounded curvature from $\omega_{S}$ to $\omega_{T}$. Indeed, $\vec{U}_{S}$ (resp. $\vec{U}_{T}$ ) is the vector tangent to $\mathcal{P}^{\prime \prime \prime}$ at $S$ (resp. $T$ ), by construction, and $\mathcal{P}^{\prime \prime \prime}$ is a path of bounded curvature since $\mathcal{R}_{S}$ and $\mathcal{R}_{T}$ and all the obstacles of $\Omega$ are moderate.

If $I=S$, the orientation of $\mathcal{P}$ at $I$ can only be $\vec{U}_{S}$ or $-\vec{U}_{S}$ since $I$ is the last intersection point between $\mathcal{P}$ and $C_{L}(S) \cup C_{R}(S)$. But only the latter cases can occur since otherwise, $\mathcal{P}$ would not be optimal. As by definition, $I$ lies before $I^{\prime}$ along $\mathcal{P}$, the part of $\mathcal{P}$ from $\left(S, \vec{U}_{S}\right)$ to $I^{\prime}$ is longer than the shortest Dubins' path from $\left(S, \vec{U}_{S}\right)$ to $\left(S,-\vec{U}_{S}\right)$ which is a path of type $C C C$ of length $2 \pi+\pi / 3$. Let $S I^{\prime}$ be the concatenation of the $\operatorname{arc} S A$ of $C_{L}(S)$ and the circular arc $A I^{\prime}$, and let $\mathcal{P}^{\prime}$ be the concatenation of $S I^{\prime}$ and the part of $\mathcal{P}$ from $I^{\prime}$ to $T$. As, the length of $S I^{\prime}$ is at most $2 \pi, \mathcal{P}^{\prime}$ is shorter than $\mathcal{P}$. We then get a contradiction as above.

Similar arguments hold if $\mathcal{P}$ also intersects $\mathcal{R}_{T}$.

Theorem 1. If $\mathcal{R}_{S}$ and $\mathcal{R}_{T}$ are disjoint and do not intersect the obstacles $\Omega$, then $\mathcal{P}$ is an Euclidean shortest path from $S$ to $T$ avoiding $\Omega$ and the two additional obstacles $\mathcal{R}_{S}$ and $\mathcal{R}_{T}$.

Proof. It follows from Lemma 1 that a SBC path from $\omega_{S}$ to $\omega_{T}$ in the presence of the obstacles $\Omega$ is also a SBC path in the presence of the obstacles $\Omega, \mathcal{R}_{S}$ and $\mathcal{R}_{T}$. On the other hand, an Euclidean shortest path from $S$ to $T$ avoiding $\Omega, \mathcal{R}_{S}$ and $\mathcal{R}_{T}$ is a path of bounded curvature from $\omega_{S}$ to $\omega_{T}$. Thus, $\mathcal{P}$ is an Euclidean shortest path from $S$ to $T$ in the presence of the obstacles $\Omega, \mathcal{R}_{S}$ and $\mathcal{R}_{T}$.

Corollary 1. A Dubins' path of type CCC between two configurations $\omega_{S}$ and $\omega_{T}$ is optimal only if the two regions $\mathcal{R}_{S}$ and $\mathcal{R}_{T}$ intersect.

In the rest of the paper, we will assume that Theorem 1 does not apply.

\section{Characterization of the $C$-segments}

We first recall the following lemma mentioned in the introduction which follows from Refs. 12 or 5 :

Lemma 2. Each subpath of an optimal path which has no point in common with the obstacles except possibly its two end points must be of type CCC or CSC.

We now recall three lemmas and Theorem 2 which were established by Agarwal et al. ${ }^{1}$ For completeness, we give the proofs (in our more restricted case of moderate obstacles). 
Lemma 3. Any non-terminal C-segment of an optimal path is longer than $\pi$.

Proof. Because the obstacles are moderate, no obstacle can touch the inner side of the $C$-segment (otherwise the $C$-segment would be an $O$-segment). Moreover, since the $C$-segment is preceded and followed by some arcs, the path can be shortened using a circular arc of radius greater than 1 (see Figure 2a).

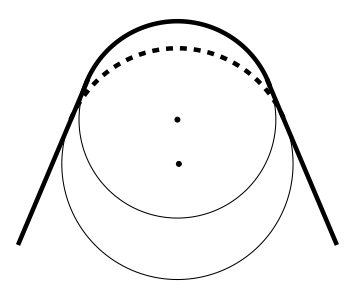

(a)

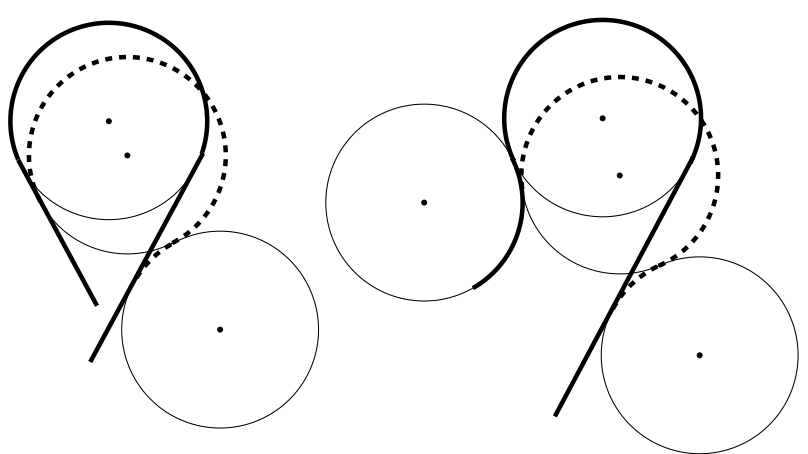

(b)

(c)

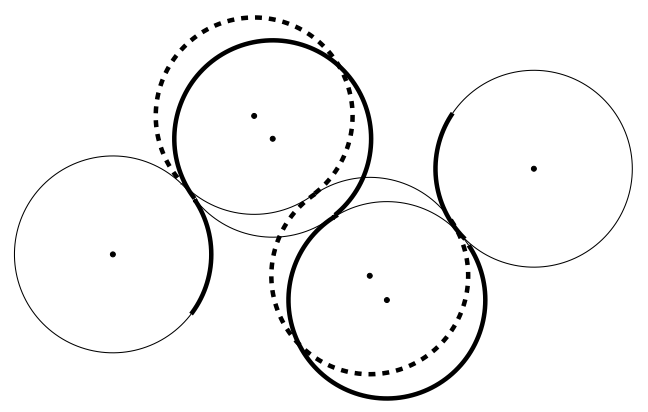

(d)

Fig. 2. Dubins' length-reducing perturbations.

Lemma 4. Any optimal path does not contain a subpath of type CCC, except when the first or the last $C$-segment of this subpath is terminal.

Proof. Assume for a contradiction that none of the $C$-segments is terminal. By Lemma 3, the length of each $C$-segment is greater than $\pi$. Therefore, the middle $C$ segment together with some portions of the other two $C$-segments can be replaced by a shortcut which cannot be obstructed by any moderate obstacle (see Figure 3). This contradicts the hypothesis and proves that one of the $C$-segments is terminal. $\square$ 


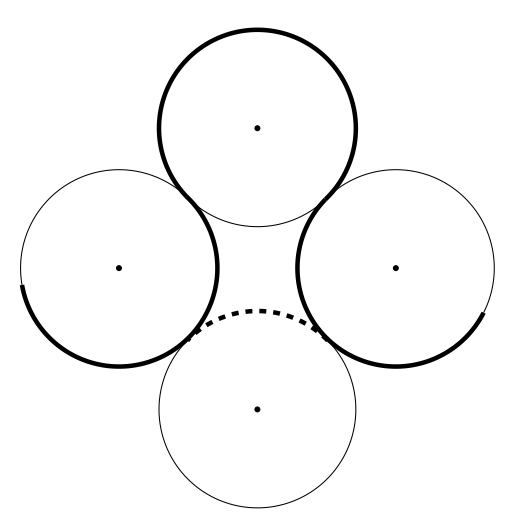

Fig. 3. Shortcut of a subpath of type $C C C$ (Lemma 4).

Lemma 5. If an optimal path contains a subpath of type $X C Y$, where $X, Y \in$ $\left\{S, O, C_{t}\right\}$, then the $C$-segment is anchored.

Proof. By Lemma 3, the $C$-segment is longer than $\pi$. According to Dubins ${ }^{12}$, perturbations (b) and (c) of Figure 2 shorten paths of type $S C S$ and $C C S$ in an obstacle-free environment. The result follows since the $O$-segments are either line segments or circular arcs of unit radius.

Theorem 2. Any C-segment appearing in an optimal path belongs to one of the following subpaths:

$$
\overline{\bar{C}}, \quad \bar{C} \bar{C}, C_{t} C \bar{C}, \quad \bar{C} C C_{t} .
$$

Proof. A $C$-segment is either terminal or belongs to a subpath of one of the three types $X C Y, X C C Y, C C C$ where $X, Y \in\{S, O\}$. If the subpath is of type $X C Y$, the $C$-segment is anchored by Lemma 5 . If the subpath is of type $X C C Y$, both $C$-segments must be tangent to some obstacle, due to Lemma 2. If the subpath is of type $C C C$, then, by Lemma 4 , the first or the third $C$-segment must be terminal. Then, assume without loss of generality that the subpath is of type $C_{t} C C$. If the next segment is a $C$-segment, it is terminal by Lemma 4 and one of the two intermediate $C$-segments must touch an obstacle by Lemma 2 . If the next segment is not a $C$-segment, then the last $C$-segment must touch an obstacle by Lemma 2 .

We now show that the possible types of $C$-segments that can appear in an optimal path can be further restricted.

Lemma 6. In an obstacle-free environment, the two perturbation shown in Figure 4 shorten a path of type CCCS such that the lengths of its second and third $C$ segments are greater than $\pi$. 


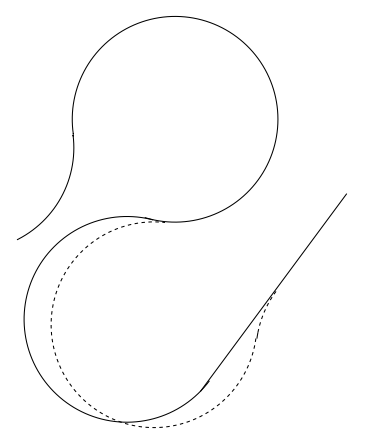

(a)

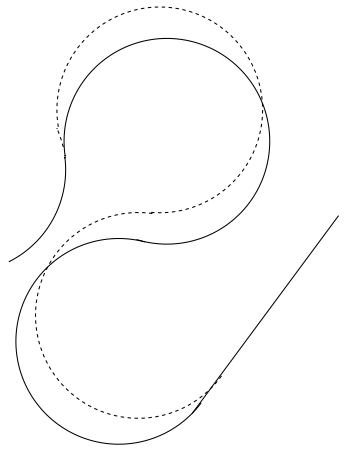

(b)

Fig. 4. Length reducing perturbations for $C C C S$ paths.

Proof. The perturbation (a) in Figure 4, similarly as the perturbation shown in Figure 2c, has been proved to shorten the path. ${ }^{12}$ We now prove that perturbation (b) in Figure 4 shortens the path.

We consider, without loss of generality, that the straight line segment of the path belongs to the $x$-axis and that the center of the circle supporting the first $C$-segment belongs to the $y$-axis (see Figure 5).

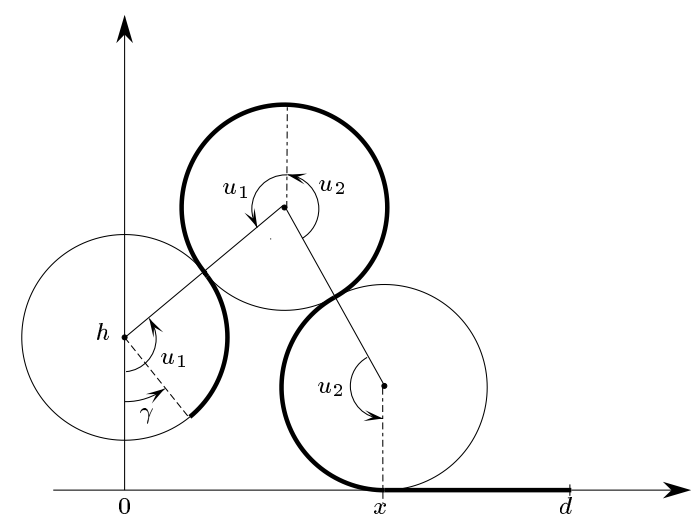

Fig. 5. Study of the perturbation (b) in Figure 4 ( $u_{2}$ is here smaller than $\pi$ for the sake of clarity).

With the notations of Figure 5, the length $L$ of a CCCS path is equal, modulo $2 \pi$, to $2\left(u_{1}+u_{2}\right)-\gamma+d-x$ where $u_{1} \in[0,2 \pi)$, the lengths $\left(u_{1}+u_{2}\right)$ and $u_{2}$ of the second and third $C$-segments belong to $(\pi, 2 \pi)$, and where $\gamma$ and $d$ are some 
constants. Furthermore, we have:

$$
\left\{\begin{array}{l}
\sin \left(u_{1}\right)+\sin \left(u_{2}\right)=x / 2 \\
\cos \left(u_{1}\right)-\cos \left(u_{2}\right)=(h-1) / 2
\end{array}\right.
$$

We compute the derivative of each equation with respect to $x$ and solve the system. We obtain the following solution $\left(\sin \left(u_{1}+u_{2}\right) \neq 0\right.$ since $\left.\left(u_{1}+u_{2}\right) \in(\pi, 2 \pi)\right)$ :

$$
\left\{\begin{array}{l}
\frac{\partial u_{1}}{\partial x}=\frac{\sin \left(u_{2}\right)}{2 \sin \left(u_{1}+u_{2}\right)} \\
\frac{\partial u_{2}}{\partial x}=\frac{\sin \left(u_{1}\right)}{2 \sin \left(u_{1}+u_{2}\right)}
\end{array}\right.
$$

Therefore,

$$
\frac{\partial L}{\partial x}=\frac{\sin \left(u_{1}\right)+\sin \left(u_{2}\right)}{\sin \left(u_{1}+u_{2}\right)}-1=\frac{\cos \left(\frac{u_{1}-u_{2}}{2}\right)-\cos \left(\frac{u_{1}+u_{2}}{2}\right)}{\cos \left(\frac{u_{1}+u_{2}}{2}\right)} .
$$

$\cos \left(\frac{u_{1}+u_{2}}{2}\right)<0$ since $\left(u_{1}+u_{2}\right) \in(\pi, 2 \pi)$. Moreover, since $u_{1} \geq 0$ and $u_{2}>0$,

$$
-\pi<-\frac{u_{1}+u_{2}}{2} \leq \frac{u_{1}-u_{2}}{2}<\frac{u_{1}+u_{2}}{2}<\pi
$$

and thus $\cos \left(\frac{u_{1}+u_{2}}{2}\right) \leq \cos \left(\frac{u_{1}-u_{2}}{2}\right)$, with equality only if $u_{1}=0$. Thus, $\frac{\partial L}{\partial x} \leq 0$, with equality only if $u_{1}=0$, which corresponds to only one value of $x$. Therefore, the perturbation shown in Figure $4 \mathrm{~b}$ shortens the path.

Lemma 7. An optimal path of type $C_{t} C C X$, where $X$ is an $O, S$ or $C$-segment, is necessarily of type $C_{t} \overline{\bar{C}} C C_{t}, C_{t} \overline{\bar{C}} \bar{C} Y$ where $Y \in\{O, S\}$, or $C_{t} C \overline{\bar{C}} Z$ where $Z \in$ $\left\{O, S, C_{t}\right\}$.

Proof. We consider first the case where $X$ is a circular arc (a $C$-segment or an $O$ segment). Then, by Lemma $4, X$ is either a terminal $C$-segment or an $O$-segment. We consider the same perturbation that Dubins used to reduce the length of paths of type $C C C C$ in an obstacle-free environment (see Figure $2 \mathrm{~d}$ and Refs. 12 or 5 for a proof). It follows from the optimality of the path, that the second or the third $C$-segment of the path of type $C_{t} C C X$ is clamped by an obstacle.

If second $C$-segment is clamped by an obstacle, then, since it is also adjacent to a terminal $C$-segment, it is anchored. Thus the path is of type $C_{t} \overline{\bar{C}} C X$ where $X \in\left\{C_{t}, O\right\}$. It follows that the path is of type $C_{t} \overline{\bar{C}} C C_{t}$ or $C_{t} \overline{\bar{C}} \bar{C} O$.

Now, if the third $C$-segment of the path of type $C_{t} C C X$ is clamped by an obstacle, then since it is also adjacent to a terminal $C$-segment or to an $O$-segment, it is anchored. Thus, the path is of type $C_{t} C \overline{\bar{C}} X$ where $X \in\left\{C_{t}, O\right\}$.

We now consider the case where $X$ is a $S$-segment; the path is of type $C_{t} C C S$. Then, by Lemma 6 , the third $C$-segment is anchored or both the second and the third $C$-segments are tangent to some obstacles. In the first case, the path is of type $C_{t} C \overline{\bar{C}} S$. In the second case, the path is of type $C_{t} \overline{\bar{C}} \bar{C} S$ because the second $C$-segment is anchored since it is tangent to an obstacle and adjacent to a terminal $C$-segment. 
Therefore, the path is of type $C_{t} \overline{\bar{C}} C C_{t}, C_{t} \overline{\bar{C}} \bar{C} O, C_{t} C \overline{\bar{C}} X$ where $X \in\left\{C_{t}, O\right\}$, $C_{t} C \overline{\bar{C}} S$ or $C_{t} \overline{\bar{C}} \bar{C} S$. Hence, the type is $C_{t} \overline{\bar{C}} C C_{t}, C_{t} \overline{\bar{C}} \bar{C} Y$ where $Y \in\{O, S\}$, or $C_{t} C \overline{\bar{C}} Z$ where $Z \in\left\{O, S, C_{t}\right\}$.

Theorem 3. Any C-segment of an optimal path belongs to one of the following subpaths:

$$
\overline{\bar{C}}, \bar{C} \bar{C}, C_{t} C \overline{\bar{C}}, \overline{\bar{C}} C C_{t}
$$

Proof. By Theorem 2, we only have to consider subpaths of type $C_{t} C \bar{C}$ or $\bar{C} C C_{t}$ where the $\bar{C}$-segment is not terminal. Without loss of generality, we consider a subpath of type $C_{t} C \bar{C}$. Since the $\bar{C}$-segment is not terminal, the subpath is part of an optimal path of type $C_{t} C \bar{C} X$ where $X$ is an $O, S$ or $C$-segment. The result then follows from Lemma 7 because a path of type $C_{t} \overline{\bar{C}} \bar{C} Y, Y \in\{O, S\}$, can be decomposed in three subpaths of type $\overline{\bar{C}}, \bar{C} \bar{C}$ and $Y$.

For a given set of obstacles, the number of anchored circles is finite, thus, the number of possible subpaths in Theorem 3 is finite except for the subpaths of type $\bar{C} \bar{C}$. The two following sections show that these subpaths can be reduced to a finite set of candidates. First, in Section 4, we show that any non-terminal subpath of type $\bar{C} \bar{C}$ of an optimal path is necessarily contained in a subpath of type $X S \bar{C} \bar{C} S X^{\prime}$ where $X, X^{\prime} \in\{O, \overline{\bar{C}}\}$. Then, in Section 5, we show that, given $X, X^{\prime} \in\{O, \overline{\bar{C}}\}$ and two obstacle edges, we can compute a finite family of candidate paths of type $X S \bar{C} \bar{C} S X^{\prime}$, where the $\bar{C}$-segments are tangent to the given obstacle edges.

\section{Characterization of the subpaths of type $\bar{C} \bar{C}$}

We prove in this section the following theorem.

Theorem 4. Any non-terminal subpath of type $\bar{C} \bar{C}$ of an optimal path is necessarily contained in a subpath of type $X S \bar{C} \bar{C} S X^{\prime}$ where $X, X^{\prime} \in\{O, \overline{\bar{C}}\}$ and where the length of the $S$-segments can be equal to zero.

We introduce the following notations. For a given subpath $\mathcal{P}$, let $C_{i}$ denote the $i$-th $C$-segment of $\mathcal{P}, \mathcal{C}_{i}$ the circle supporting $C_{i}$, and $O_{i}$ the center of $\mathcal{C}_{i}$.

We first establish three lemmas and a proposition.

Lemma 8. In a subpath of type CCS of an optimal path, such that the first $C$ segment is not terminal, the length of the $S$-segment is smaller than $4 \cos \alpha$, where $\alpha=\angle\left(\overrightarrow{O_{2} O_{1}}, \vec{u}\right)$ and $\vec{u}$ is the direction of the $S$-segment (see Figure 6$)$.

Proof. Let $\mathcal{P}$ be the optimal subpath of type $C C S$. Since the length of each $C$ segment is strictly greater than $\pi$ and smaller than $2 \pi, \alpha$ belongs to $(-\pi / 2, \pi / 2)$ (see Figure 6). We distinguish three cases according to the value of $\alpha$ and, in each case, we exhibit a shortcut that cannot be intersected by a moderate obstacle. Let $\mathcal{S}$ denote the line supporting the $S$-segment. 
1. $\alpha \in(0, \pi / 2)$ (Figure 6a): if the length of the $S$-segment is greater or equal to $4 \cos \alpha$, the dashed $C$-segment shortens $\mathcal{P}$.

2. $\alpha \in(-\pi / 2,-\pi / 3)$ (Figure $6 \mathrm{~b}$ ): let $\mathcal{C}^{\prime}$ be the circle (of unit radius) tangent to $\mathcal{C}_{1}$ and $\mathcal{S}$, and lying on the same side of $\mathcal{S}$ as $\mathcal{C}_{2}$. Since $|\alpha|>\pi / 3, \mathcal{C}^{\prime}$ intersects $\mathcal{C}_{2}$ and therefore the length of the dashed $C$-segment on $\mathcal{C}_{1}$ is smaller than $\pi / 3$. Moreover, the length of the dashed $C$-segment on $\mathcal{C}^{\prime}$ is smaller than $\pi / 2$ because the line segment intersects $\mathcal{C}_{1}$. It follows that the length of the dashed path is smaller than $\pi$ and so is smaller than the length of the $C$-segment $C_{2}$. Hence, if the length of the $S$-segment is greater or equal to $4 \cos \alpha$, the dashed path shortens $\mathcal{P}$.

3. $\alpha \in[-\pi / 3,0]$ (Figures $6 \mathrm{c}$ and $6 \mathrm{~d}$ ): if the length of the $S$-segment is greater or equal to 2, let $A, B, C, D$ and $I$ be the points of $\mathcal{P}$ as defined in Figures $6 \mathrm{c}$ and $6 \mathrm{~d}$. Let $\mathcal{P}^{\prime}$ be the path obtained from $\mathcal{P}$ by replacing the part of $\mathcal{P}$ from $A$ to $D$ by the (dashed) circular arc $A B$, followed by the long circular arc $B C$ of $\mathcal{C}_{2}$, followed by the (dashed) circular $\operatorname{arc} C D$ (see Figures $6 \mathrm{c}$ and $6 \mathrm{~d}$ ). The length of $\mathcal{P}^{\prime}$ minus the length of $\mathcal{P}$ is equal to the sum of the lengths of the circular arcs $A B$ and $C D$ minus the length of the parts of $\mathcal{P}$ between $A$ and $C$, and between $B$ and $D$. This difference is negative because the length of the circular arc $A B$ is smaller than the sum of the lengths of the two $\operatorname{arcs} A I$ and $B I$ of $\mathcal{P}$ (because $A I$ and $B I$ are outside the circle supporting the $\operatorname{arc} A B$ ), and similarly, the length of the circular $\operatorname{arc} C D$ is smaller than the sum of the lengths of the two $\operatorname{arcs} I C$ and $I D$ of $\mathcal{P}$. Thus, if the length of the $S$-segment is greater or equal to 2 , path $\mathcal{P}$ is not optimal. Thus, the length of the $S$-segment is smaller than 2, which is smaller or equal to $4 \cos \alpha$ for any $\alpha \in[-\pi / 3,0]$.

We now consider subpaths of type $C C S C$.

Lemma 9. Let $\mathcal{P}$ be a subpath of type CCSC of an optimal path, such that the first and the last $C$-segments are not terminal. If the two $C$-segments $C_{2}$ and $C_{3}$ that are adjacent to the $S$-segment have the same orientation (resp. opposite orientations), the distance between $O_{1}$ and $O_{3}$ is less than 2 (resp. 4).

Proof. By the previous lemma, the length $s$ of the $S$-segment is less than $4 \cos \alpha$. If $\mathcal{C}_{2}$ and $\mathcal{C}_{3}$ have the same orientation,

$$
O_{1} O_{3}^{2}=\left(\overrightarrow{O_{1} O_{2}}+\overrightarrow{O_{2} O_{3}}\right)^{2}=4+s^{2}-4 s \cos \alpha<4 .
$$

If the two circles have opposite orientations, let $O_{3}^{\prime}$ be the point symmetric to $O_{3}$ with respect to the $S$-segment. The length of $O_{1} O_{3}$ is less than the sum of the length of $O_{3} O_{3}^{\prime}$, which is equal to 2, and of the length of $O_{1} O_{3}^{\prime}$, which is less than 2 by the above inequality.

Lemma 10. In a subpath of type CCSC of an optimal path, such that the first and the last $C$-segments are not terminal, the two $C$-segments adjacent to the $S$-segment 


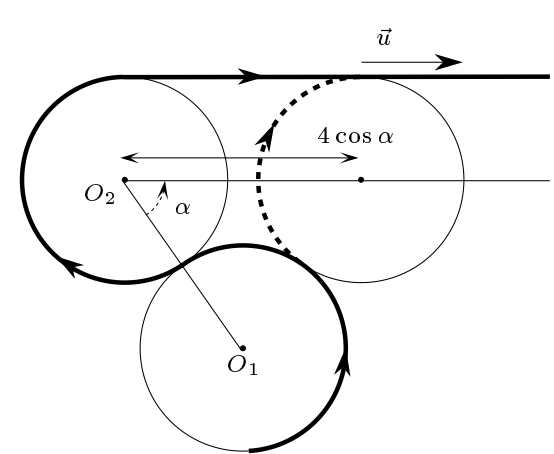

(a): $\alpha \in(0, \pi / 2)$

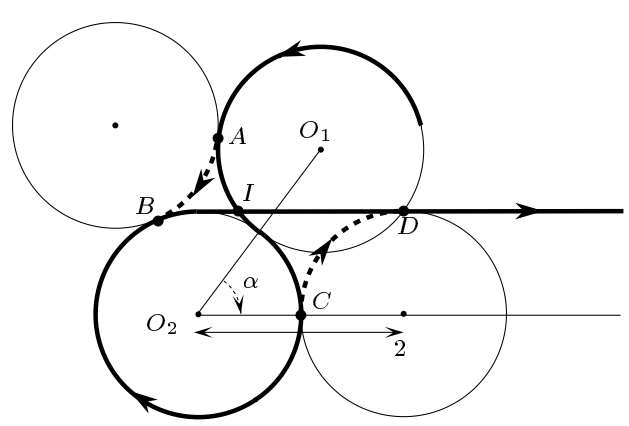

(c): $\alpha \in(-\pi / 3,-\pi / 6)$

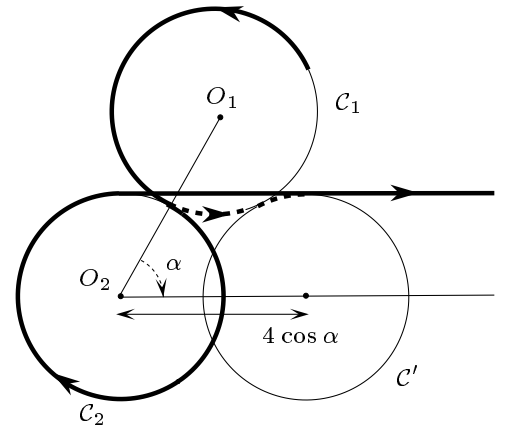

(b) : $\alpha \in(-\pi / 2,-\pi / 3)$

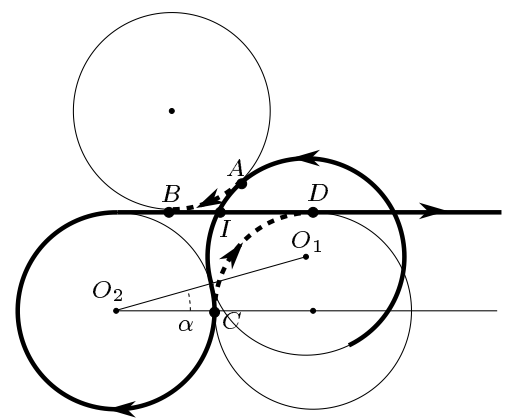

(d): $\alpha \in(-\pi / 6,0)$

Fig. 6. Shortcuts used in Lemma 8.

have the same orientation (clockwise or counterclockwise).

Proof. We consider a subpath $\mathcal{P}$ of type $C C S C$ of an optimal path where $C_{1}$ and $C_{3}$ are not terminal and such that $C_{2}$ and $C_{3}$ have opposite orientations (see Figure 7 ). We show that such a path can be shortened. The previous lemma implies that there exists a circle of unit radius tangent to the circles $\mathcal{C}_{1}$ and $\mathcal{C}_{3}$.

Suppose first that the circles $\mathcal{C}_{1}$ and $\mathcal{C}_{3}$ do not intersect (see Figure 7). Since the lengths of $C_{1}$ and $C_{3}$ are greater than $\pi$, there exists a $C$-segment of length smaller than $\pi$ tangent to both $C_{1}$ and $C_{3}$. This $C$-segment clearly shortens $\mathcal{P}$ and avoids the moderate obstacles.

Suppose now that $\mathcal{C}_{1}$ and $\mathcal{C}_{3}$ intersect. The previous argument does not hold since there does not necessarily exist a circle tangent to the $C$-segments $C_{1}$ and 
$C_{3}$ (see Figure 8a). However, the shortcut shown in Figure 8 shortens $\mathcal{P}$ since the length of the dashed $C$-segment $A B$ is shorter than the sum of the lengths of the $\operatorname{arcs} A I$ and $B I$ of $\mathcal{P}$, and, similarly, the length of the dashed $C$-segment $C D$ is shorter than the sum of the lengths of the $\operatorname{arcs} I C$ and $I D$.

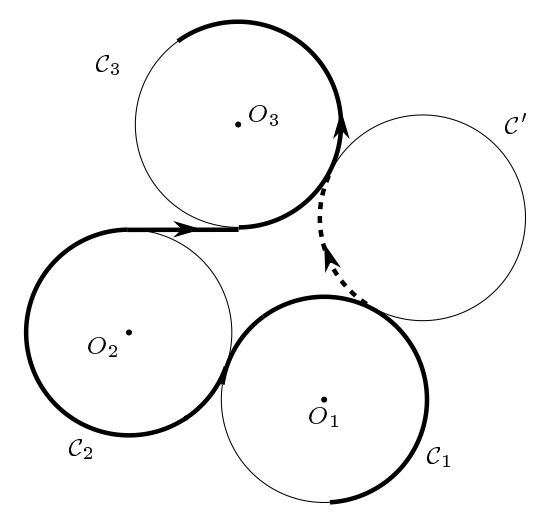

Fig. 7. Shortcut used in Lemma 10 if $\mathcal{C}_{1}$ does not intersect $\mathcal{C}_{3}$.

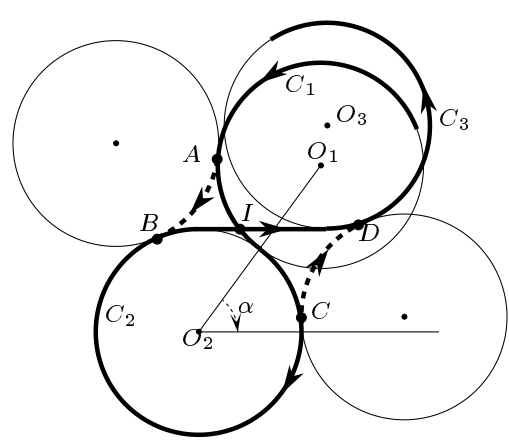

(a): $\alpha \in(-\pi / 2,-\pi / 6)$

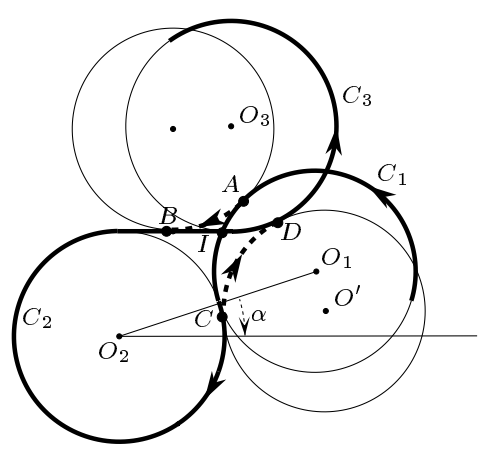

(b): $\alpha \in(-\pi / 6,0)$

Fig. 8. Shortcut used in Lemma 10 if $\mathcal{C}_{1}$ does intersect $\mathcal{C}_{3}$.

Proposition 1. An optimal path cannot contain a subpath of type CCSCC, except when the first or the last C-segment of this subpath is terminal.

Proof. Assume for a contradiction that an optimal path contains a nonterminal subpath of type $C C S C C$. By Lemma 3 , the lengths of $C_{1}$ and $C_{4}$ are greater than $\pi$. According to the previous lemma, both $C$-segments $C_{2}$ and $C_{3}$ have the same 
orientation. We assume, without loss of generality, that the first $C$-segment $C_{1}$ is oriented counterclockwise and show that the dashed $C$-segment tangent to $C_{1}$ and $C_{4}$, shown in Figure 9, shortens the path.

Let $M_{12}$ be the common end point of $C_{1}$ and $C_{2}$, and $M_{34}$ the common end point of $C_{3}$ and $C_{4}$. Let $\alpha$ and $\beta$ be defined as in Figure 9 and let $s$ be the length of the $S$-segment.

We now show that there exists a $C$-segment of length smaller than $\pi$ oriented clockwise and tangent to $C_{1}$ and $C_{4}$. By Lemma 9, the length $O_{2} O_{4}$ is less than 2, implying that the length $O_{1} O_{4}$ is less than 4 . Thus there exist two circles of unit radius tangent to $\mathcal{C}_{1}$ and $\mathcal{C}_{4}$. Since the length $O_{2} O_{4}$ is less than 2, $M_{12}$ belongs to the dashed $C$-segment between $A$ and $B$ in Figure 10 . Since $C_{1}$ is oriented counterclockwise and its length is greater than $\pi$, point $B$ belongs to $C_{1}$ (see Figure 10). Similarly, point $D$ belongs to $C_{4}$. Moreover, the $C$-segment oriented clockwise and tangent to $\mathcal{C}_{1}$ at $B$ and to $\mathcal{C}_{4}$ at $D$ is smaller than $\pi$. It follows that this $C$-segment shortens the subpath of type CCSCC. This concludes the proof because such a $C$-segment avoids any moderate obstacle.

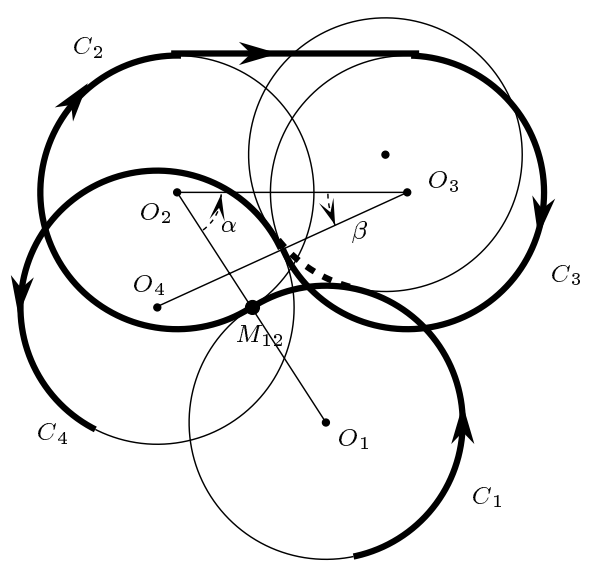

Fig. 9. Shortcut used in Proposition 1.

Proof of Theorem 4: Considering that the length of the $S$-segments can be equal to zero, a non-terminal subpath of type $\bar{C} \bar{C}$ is necessarily contained in a subpath of type $X S \bar{C} \bar{C} S X^{\prime}$ where $X, X^{\prime} \in\{O, C\}$. We want to prove that $X, X^{\prime} \in\{O, \overline{\bar{C}}\}$. Assume for a contradiction that $X \notin\{O, \bar{C}\}$ (the proof for $X^{\prime}$ is similar). Then, $X$ is a non-terminal $C$-segment and Lemma 4 implies that the length of the first $S$-segment of the subpath is strictly positive. Thus, by Theorem 3, $X$ is necessarily a $C$-segment following another $C$-segment, noted $Y$. Then, by Proposition 1, $Y$ is terminal and thus, by Lemma 5, $X$ is an anchored $C$-segment. This contradicts our assumption and ends the proof of the theorem. 


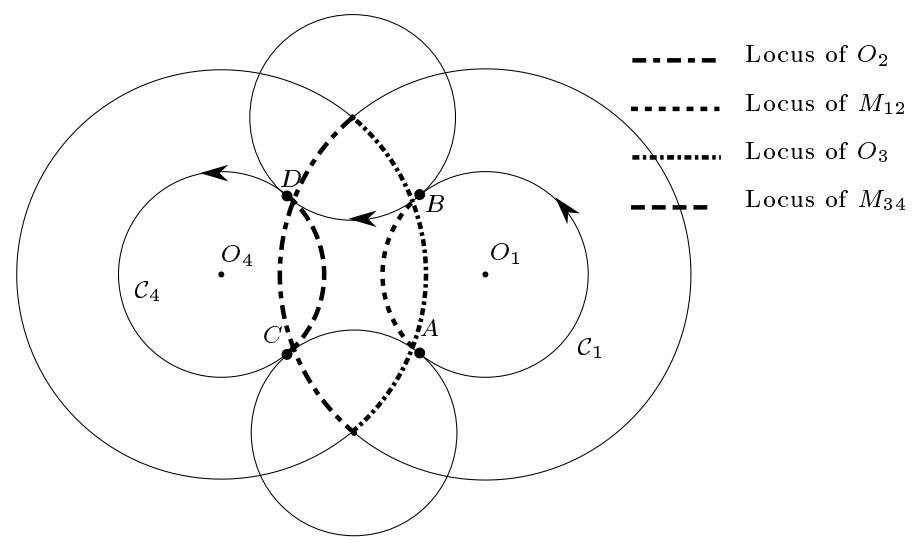

Fig. 10. For the proof of Proposition 1.

\section{Bounding the number of subpaths of type $\bar{C} \bar{C}$}

This section is devoted to the proof of the following theorem. Let $\mathcal{X}$ be a circular edge of some obstacle or an anchored circle. Let $\mathcal{X}^{\prime}$ be another such circular arc and let $\mathcal{O}$ and $\mathcal{O}^{\prime}$ be two obstacle edges. Let $X$ denote an $\operatorname{arc}$ of $\mathcal{X}, X^{\prime}$ an $\operatorname{arc}$ of $\mathcal{X}^{\prime}, \bar{C}$ a $C$-segment tangent to $\mathcal{O}$, and $\bar{C}^{\prime}$ a $C$-segment tangent to $\mathcal{O}^{\prime}$.

Theorem 5. We can compute in $O(1)$ time a family of $O(1)$ candidate paths such that any optimal path of type $X S \bar{C} \bar{C}^{\prime} S X^{\prime}$, where neither $\bar{C}$ nor $\bar{C}^{\prime}$ is anchored nor the length of the $S$-segments is equal to zero, is contained in one of the candidate paths.

Proof. Let $\mathcal{P}$ denote a path of type $X S \bar{C} \bar{C}^{\prime} S X^{\prime}$ and assume that neither $\bar{C}$ nor $\bar{C}^{\prime}$ is anchored when path $\mathcal{P}$ is optimal.

There exists at most one path $\mathcal{P}$ such that the first $S$-segment is reduced to a point unless $\mathcal{X}$ and $\mathcal{O}$ coincide. If $\mathcal{X}$ and $\mathcal{O}$ coincide and if $\mathcal{P}$ is of type $X \bar{C} \bar{C}^{\prime} S X^{\prime}$, then, by Lemma $7, \bar{C}$ or $\bar{C}^{\prime}$ is anchored which implies that $\mathcal{P}$ is not optimal. Thus there exists at most one possibly optimal path $\mathcal{P}$ such that the first $S$-segment is reduced to a point, and similarly for the last $S$-segment. We consider as candidate path these two paths, if they exists. We can thus assume in the following that none of the $S$-segments is reduced to a point in an optimal path $\mathcal{P}$.

We consider in turn tree cases depending on the types of the obstacle edges $\mathcal{O}$ and $\mathcal{O}^{\prime}$. We consider first the case where both $\mathcal{O}$ and $\mathcal{O}^{\prime}$ are line segments, then the case where both are circular arcs, and finally the case where one is a line segment and the other a circular arc.

In the first (resp. second, third) case, we show, in Lemma 12 (resp. 15, 18), that path $\mathcal{P}$ is optimal only if System (2) (resp. (9), (20)) is satisfied. We then show, in 
Lemma 13 (resp. 16, 19), that this system admits a finite set of solutions, or more precisely that some of the variables take finitely many distinct values in the set of solutions. Furthermore, we show in Lemma 14 (resp. 17, 20), that each of these values determines $O(1)$ paths of type $X S \bar{C} \bar{C}^{\prime} S X^{\prime}$ (where $X$ and $X^{\prime}$ are maximal). These paths are the candidate paths. Finally, since System (2) (resp. (9), (20)) is of bounded degree, its $O(1)$ solutions and the corresponding candidate paths can be computed in $O(1)$ time.

We prove, in the rest of the section, the lemmas used in the proof of Theorem 5 , namely Lemmas 12 to 20 . In the following, $\mathcal{P}$ still denotes a path of type $X S \bar{C} \bar{C}^{\prime} S X^{\prime}$ and we assume that, when $\mathcal{P}$ is optimal, neither $\bar{C}$ nor $\bar{C}^{\prime}$ is anchored and that none of the $S$-segments is reduced to a point.

We introduce the following notations (see Figures 11,12, 15 or 18). Let $\mathcal{C}$ and $\mathcal{C}^{\prime}$ be the circles supporting $\bar{C}$ and $\bar{C}^{\prime}$, and $I$ and $I^{\prime}$ their centers. Let $H$ and $H^{\prime}$ be the centers of the circles supporting $\mathcal{X}$ and $\mathcal{X}^{\prime}$. In addition, let $\alpha=\angle\left(\vec{R}, \overrightarrow{I I^{\prime}}\right)$, $\alpha^{\prime}=\angle\left(\overrightarrow{R^{\prime}}, \overrightarrow{I^{\prime} I}\right), \varphi=\angle\left(\overrightarrow{I I^{\prime}}, \overrightarrow{F^{\prime}}\right)$ and $\varphi^{\prime}=\angle\left(\overrightarrow{I^{\prime} I}, \overrightarrow{F^{\prime}}\right)$. For the sake of simplicity, we suppose in our figures that path $\mathcal{P}$ is oriented counterclockwise on $\bar{C}$ and clockwise on $\bar{C}^{\prime}$ (e.g., Figures 11, 15 and 18); however, our computations are valid in the general case.

We consider a mechanical device that consists of four fixed objects and one mobile object $D$ (see Figures 11, 15 and 18). The fixed objects are the two obstacles $\mathcal{O}$ and $\mathcal{O}^{\prime}$ and the two disks of unit radius supporting $\mathcal{X}$ and $\mathcal{X}^{\prime}$. The mobile object $D$ is the union of two tangent disks (corresponding to the circles $\mathcal{C}$ and $\mathcal{C}^{\prime}$ ). We consider a rubber band of thickness zero attached on $\mathcal{X}$ and on $\mathcal{X}^{\prime}$ and passing around $\mathcal{C}$ and $\mathcal{C}^{\prime}$. We are interested in the case where the two mobile disks are tangent to the obstacles $\mathcal{O}$ and $\mathcal{O}^{\prime}$, respectively, and are not tangent to $\mathcal{X}, \mathcal{X}^{\prime}$ or to any other obstacle. The mobile object $D$ is subject to four forces $\vec{F}, \overrightarrow{F^{\prime}}, \vec{R}$ and $\overrightarrow{R^{\prime}}$ (see Figures 11, 15 and 18). $\vec{F}$ and $\overrightarrow{F^{\prime}}$ are the two forces, of equal norm $F$, exerted by the rubber band. $\vec{R}$ and $\overrightarrow{R^{\prime}}$ are the reactions of the obstacles $\mathcal{O}$ and $\mathcal{O}^{\prime}$ onto the mobile $D$.

We first establish a lemma that holds regardless of the type of the obstacle edges $\mathcal{O}$ and $\mathcal{O}^{\prime}$.

Lemma 11. Path $\mathcal{P}$ is optimal only if

$$
\sin \left(\alpha^{\prime}-\alpha\right)+\sin \alpha^{\prime} \sin (\alpha+\varphi)-\sin \alpha \sin \left(\alpha^{\prime}+\varphi^{\prime}\right)=0 .
$$

Proof. Path $\mathcal{P}$ is optimal only if the mobile $D$ is at an equilibrium that is if and only if the sum of the forces $\vec{F}, \overrightarrow{F^{\prime}}, \vec{R}, \vec{R}^{\prime}$ and the sum of their torques is zero. The sum of the forces is zero if and only if

$$
\left\{\begin{array}{l}
R \cos \alpha+F \cos \varphi=R^{\prime} \cos \alpha^{\prime}+F \cos \varphi^{\prime} \\
R \sin \alpha-F \sin \varphi=R^{\prime} \sin \alpha^{\prime}-F \sin \varphi^{\prime}
\end{array}\right.
$$




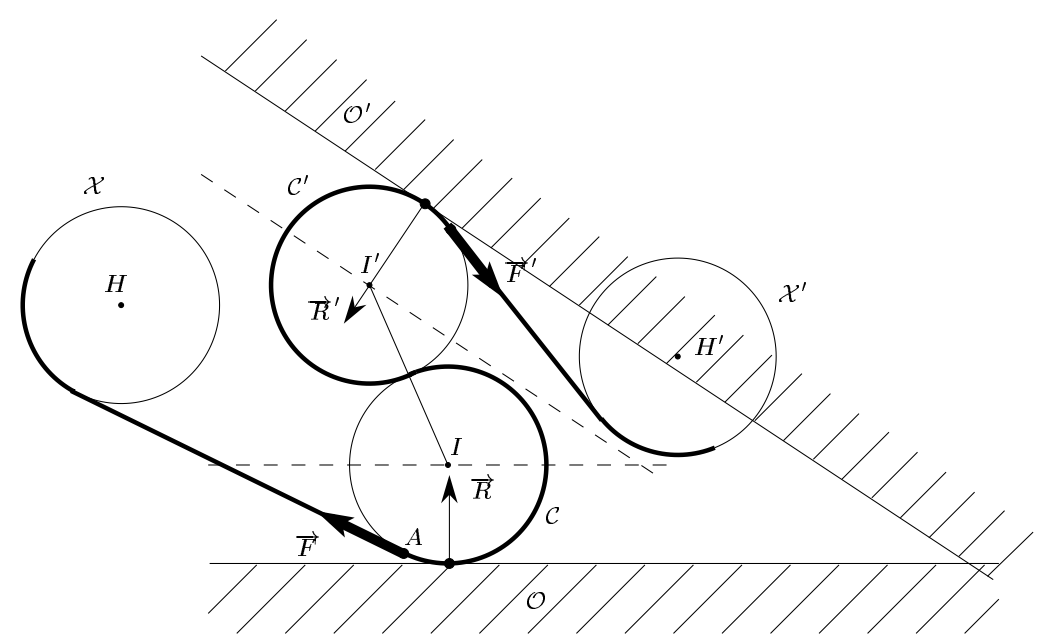

Fig. 11. The mechanical device.

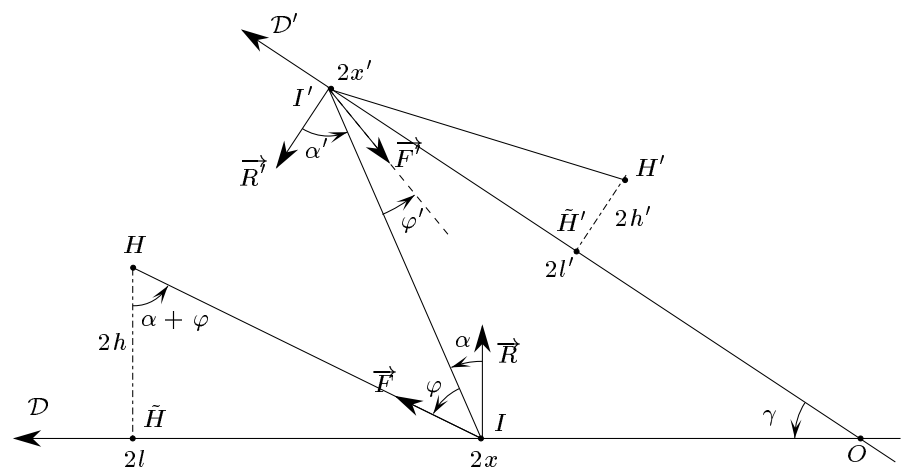

Fig. 12. For the proof of Theorem 5.

We consider the torque of the forces with respect to $I^{\prime}$; let $A$ be the supporting point of the force $\vec{F}$ on $\mathcal{C}$.

$$
\begin{aligned}
\mathcal{M}_{\vec{F} / I^{\prime}} & =\operatorname{det}\left(\vec{F}, \overrightarrow{A I^{\prime}}\right)=\operatorname{det}(\vec{F}, \overrightarrow{A I})+\operatorname{det}\left(\vec{F}, \overrightarrow{I I^{\prime}}\right) \\
& =-F-2 F \sin \varphi \\
\mathcal{M}_{F^{\prime}} / I^{\prime} & =-F \\
\mathcal{M}_{\vec{R} / I^{\prime}} & =2 R \sin \alpha \\
\mathcal{M}_{\overrightarrow{R^{\prime}} / I^{\prime}} & =0
\end{aligned}
$$

Thus the sum of these torques is zero if and only if $R \sin \alpha=F+F \sin \varphi$. Hence 
the mobile object $D$ is at an equilibrium if and only if

$$
\left\{\begin{array}{l}
R \cos \alpha+F \cos \varphi=R^{\prime} \cos \alpha^{\prime}+F \cos \varphi^{\prime} \\
R \sin \alpha-F \sin \varphi=R^{\prime} \sin \alpha^{\prime}-F \sin \varphi^{\prime} \\
R \sin \alpha=F+F \sin \varphi
\end{array}\right.
$$

We eliminate $R$ in the two first equations by multiplying the first equation by $\sin \alpha$ and replacing $R \sin \alpha$ by $F+F \sin \varphi$. We get

$$
\left\{\begin{array}{l}
F(1+\sin \varphi) \cos \alpha+F \cos \varphi \sin \alpha=R^{\prime} \cos \alpha^{\prime} \sin \alpha+F \cos \varphi^{\prime} \sin \alpha \\
F=R^{\prime} \sin \alpha^{\prime}-F \sin \varphi^{\prime} .
\end{array}\right.
$$

We now eliminate $R^{\prime}$ by summing the first equation multiplied by $\sin \alpha^{\prime}$ and the second equation multiplied by $-\cos \alpha^{\prime} \sin \alpha$. We get

$F(1+\sin \varphi) \cos \alpha \sin \alpha^{\prime}+F \cos \varphi \sin \alpha \sin \alpha^{\prime}-F \cos \alpha^{\prime} \sin \alpha=$

$$
F \cos \varphi^{\prime} \sin \alpha \sin \alpha^{\prime}+F \sin \varphi^{\prime} \cos \alpha^{\prime} \sin \alpha
$$

or equivalently

$$
\sin \left(\alpha^{\prime}-\alpha\right)+\sin \alpha^{\prime} \sin (\alpha+\varphi)-\sin \alpha \sin \left(\alpha^{\prime}+\varphi^{\prime}\right)=0 .
$$

We now study, in turn, the cases depending on whether the obstacles edges $\mathcal{O}$ and $\mathcal{O}^{\prime}$ are line segments or circular arcs.

\section{Case 1: Both obstacle edges are line segments}

Let $\mathcal{D}\left(\right.$ resp. $\mathcal{D}^{\prime}$ ) be the oriented line parallel to $\mathcal{O}\left(\mathcal{O}^{\prime}\right)$, passing through $I$ (resp. $\left.I^{\prime}\right)$ and oriented such that $\angle(\vec{R}, \mathcal{D})=\pi / 2$ (resp. $\left.\angle\left(\overrightarrow{R^{\prime}}, \mathcal{D}^{\prime}\right)=-\pi / 2\right)$ (see Figure 12).

Let $\gamma=\angle\left(\mathcal{D}^{\prime}, \mathcal{D}\right)$. Let $O$ be the intersection point between $\mathcal{D}$ and $\mathcal{D}^{\prime}(O$ is well defined since no two obstacle edges are parallel). Let $\tilde{H}$ (resp. $\tilde{H}^{\prime}$ ) be the orthogonal projection of $H$ (resp. $H^{\prime}$ ) onto $\mathcal{D}$ (resp. $\mathcal{D}^{\prime}$ ). Let $2 x$ and $2 l$ (resp. $2 x^{\prime}, 2 l^{\prime}$ ) be the algebraic lengths of $O I$ and $O \tilde{H}$ (resp. $O I^{\prime}$ and $O \tilde{H}^{\prime}$ ) on the oriented line $\mathcal{D}$ (resp. $\mathcal{D}^{\prime}$ ). Let $2 h$ be the algebraic distance between $H$ and $\mathcal{D}$ with $2 h>0$ if and only if $\overrightarrow{\tilde{H} H}$ and $\vec{R}$ have the same orientation; $2 h^{\prime}$ is defined similarly except that $2 h^{\prime}>0$ if and only if $\overrightarrow{\tilde{H}^{\prime} H^{\prime}}$ and $\overrightarrow{R^{\prime}}$ have opposite orientations.

Lemma 12. Path $\mathcal{P}$ is optimal only if

$$
\left\{\begin{array}{l}
\sin \gamma+\sin (\alpha+\gamma) \sin (\alpha+\varphi)-\sin \alpha \sin \left(\alpha^{\prime}+\varphi^{\prime}\right)=0 \\
h \sin \gamma \sin (\alpha+\varphi)-l \sin \gamma \cos (\alpha+\varphi)+\cos (\alpha+\gamma) \cos (\alpha+\varphi)+\delta \sin \gamma=0 \\
h^{\prime} \sin \gamma \sin \left(\alpha^{\prime}+\varphi^{\prime}\right)-l^{\prime} \sin \gamma \cos \left(\alpha^{\prime}+\varphi^{\prime}\right)+\cos \alpha \cos \left(\alpha^{\prime}+\varphi^{\prime}\right)+\delta^{\prime} \sin \gamma=0
\end{array}\right.
$$

where $\delta$ (resp. $\left.\delta^{\prime}\right)$ is zero if path $\mathcal{P}$ has the same orientation on $\mathcal{X}$ and $\mathcal{C}$ (resp. $\mathcal{X}^{\prime}$ and $\left.\mathcal{C}^{\prime}\right)$ and 1 otherwise.

Proof. Considering the triangle $\triangle O I I^{\prime}$ in Figure 12 yields

$$
\alpha^{\prime}=\alpha+\gamma[2 \pi]
$$


Table 1. Respective signs of $\tan (\alpha+\varphi), l-x$ and $h$.

\begin{tabular}{||c|c|c|c|c||}
\hline$\alpha+\varphi$ & $-\pi$ & $-\pi / 2$ & \multicolumn{2}{c|}{$\pi / 2$} \\
\hline $\tan (\alpha+\varphi)$ & + & - & + & - \\
\hline$l-x$ & - & - & + & + \\
\hline$h$ & - & + & + & - \\
\hline
\end{tabular}

$$
\frac{|\sin \gamma|}{2}=\frac{|\sin (\alpha+\pi / 2)|}{\left|2 x^{\prime}\right|}=\frac{\left|\sin \left(\pi / 2-\alpha^{\prime}\right)\right|}{|2 x|} .
$$

Considering the different cases that can occur, we get

$$
x=\frac{\cos \alpha^{\prime}}{\sin \gamma} \text { and } x^{\prime}=\frac{\cos \alpha}{\sin \gamma} .
$$

Eqs. (1) and (3) yield the first equation of System (2). We now show how to compute the two other equations of System (2) for each possible orientation of $X$ and $X^{\prime}$.

- $\mathcal{P}$ has the same orientation on $\mathcal{X}$ and $\mathcal{C}$.

By definition of $\alpha$ and $\varphi, \alpha+\varphi=\angle(\vec{R}, \vec{F})[2 \pi]=\angle(\overrightarrow{H \vec{H}}, \overrightarrow{H I})[\pi]$. Since $\overrightarrow{H \vec{H}}$ and $\overrightarrow{\tilde{H} I}$ are orthogonal, $\tan (\alpha+\varphi)= \pm(2 l-2 x) / 2 h$ (see Figure 12). Considering the different cases that can occur (see Table 5 and Figure 12), we get

$$
\tan (\alpha+\varphi)=\frac{l-x}{h}
$$

Using Eqs. (3) and (4), we obtain

$$
h \sin \gamma \sin (\alpha+\varphi)-l \sin \gamma \cos (\alpha+\varphi)+\cos (\alpha+\gamma) \cos (\alpha+\varphi)=0 .
$$

This equation is equal to the second equation of System (2) because $\delta=0$ in this case.

- $\mathcal{P}$ has the same orientation on $\mathcal{X}^{\prime}$ and $\mathcal{C}^{\prime}$.

Similarly as above, we get

$$
\begin{gathered}
\tan \left(\alpha^{\prime}+\varphi^{\prime}\right)=\frac{l^{\prime}-x^{\prime}}{h^{\prime}}, \\
h^{\prime} \sin \gamma \sin \left(\alpha^{\prime}+\varphi^{\prime}\right)-l^{\prime} \sin \gamma \cos \left(\alpha^{\prime}+\varphi^{\prime}\right)+\cos \alpha \cos \left(\alpha^{\prime}+\varphi^{\prime}\right)=0 .
\end{gathered}
$$

This equation is equal to the third equation of System (2) because $\delta=0$ in this case.

- $\mathcal{P}$ has opposite orientations on $\mathcal{X}$ and $\mathcal{C}$.

Let $\mu=\angle(\vec{F}, \overrightarrow{I H})$ and $\varepsilon=\angle(\overrightarrow{I H}, \mathcal{D})$ (see Figure 13). With these definitions, we have

$$
\begin{gathered}
\alpha+\varphi+\mu+\varepsilon=\pi / 2[2 \pi], \\
\sin \mu=\frac{2}{I H}, \quad \sin \varepsilon=\frac{2 h}{I H}, \quad \cos \varepsilon=\frac{2 l-2 x}{I H} .
\end{gathered}
$$



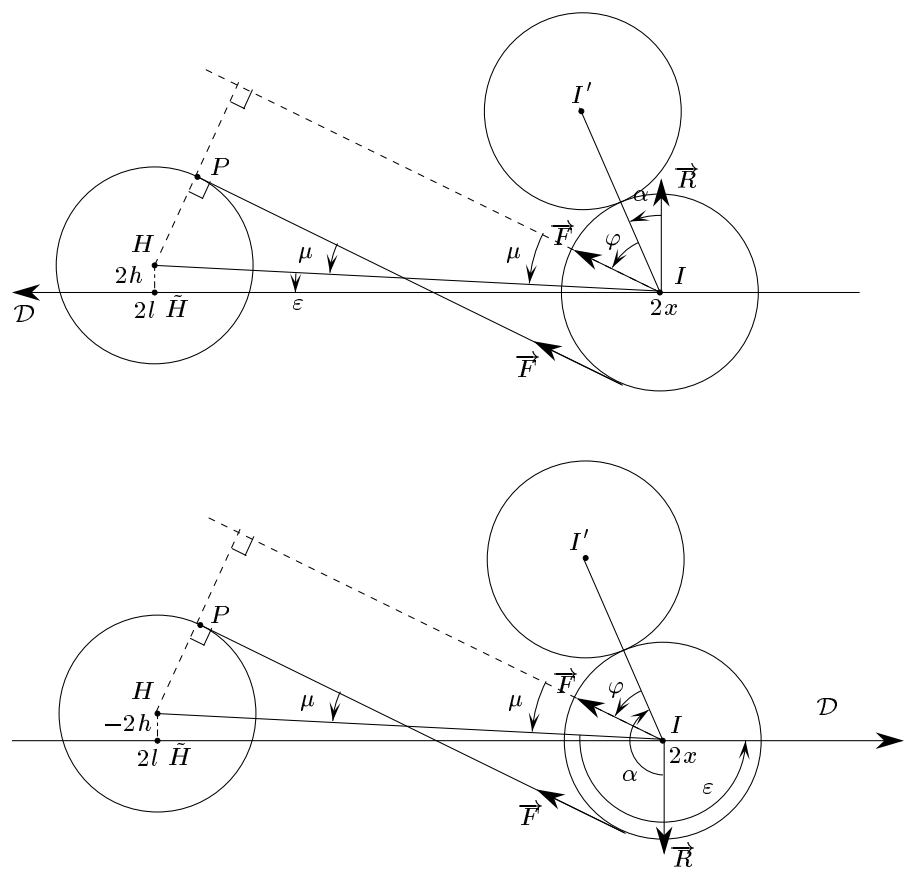

Fig. 13. For computing Eq. (6).

Therefore, $\alpha+\varphi+\varepsilon=\pi / 2-\mu[2 \pi], \cos (\alpha+\varphi) \cos \varepsilon-\sin (\alpha+\varphi) \sin \varepsilon=\sin \mu$ and

$$
(l-x) \cos (\alpha+\varphi)-h \sin (\alpha+\varphi)=1 .
$$

By Eqs. (3) and (4), we obtain

$$
h \sin \gamma \sin (\alpha+\varphi)-l \sin \gamma \cos (\alpha+\varphi)+\cos (\alpha+\gamma) \cos (\alpha+\varphi)+\sin \gamma=0 .
$$

This is equal to the second equation of System (2) because $\delta=1$ in this case.

- $\mathcal{P}$ have opposite orientations on $\mathcal{X}^{\prime}$ and $\mathcal{C}^{\prime}$.

Let $\mu^{\prime}=\angle\left(\overrightarrow{F^{\prime}}, \overrightarrow{I^{\prime} H^{\prime}}\right)$ and $\varepsilon^{\prime}=\angle\left(\overrightarrow{I^{\prime} H^{\prime}}, \mathcal{D}^{\prime}\right)$ (see Figure 14). We have

$$
\alpha^{\prime}+\varphi^{\prime}+\mu^{\prime}+\varepsilon^{\prime}=-\pi / 2[2 \pi]
$$

$$
\sin \mu^{\prime}=\frac{2}{I^{\prime} H^{\prime}}, \quad \sin \varepsilon^{\prime}=-\frac{2 h^{\prime}}{I^{\prime} H^{\prime}}, \quad \cos \varepsilon^{\prime}=\frac{2 x^{\prime}-2 l^{\prime}}{I^{\prime} H^{\prime}} .
$$

Thus, $\alpha^{\prime}+\varphi^{\prime}+\varepsilon^{\prime}=-\pi / 2-\mu^{\prime}, \cos \left(\alpha^{\prime}+\varphi^{\prime}\right) \cos \varepsilon^{\prime}-\sin \left(\alpha^{\prime}+\varphi^{\prime}\right) \sin \varepsilon^{\prime}=-\sin \mu^{\prime}$ and

$$
\left(x^{\prime}-l^{\prime}\right) \cos \left(\alpha^{\prime}+\varphi^{\prime}\right)+h^{\prime} \sin \left(\alpha^{\prime}+\varphi^{\prime}\right)=-1 .
$$

Then, by Eq. (4)

$$
h^{\prime} \sin \gamma \sin \left(\alpha^{\prime}+\varphi^{\prime}\right)-l^{\prime} \sin \gamma \cos \left(\alpha^{\prime}+\varphi^{\prime}\right)+\cos \alpha \cos \left(\alpha^{\prime}+\varphi^{\prime}\right)+\sin \gamma=0 .
$$

This equation is equal to the third equation of System (2) because $\delta=1$ in this case. 

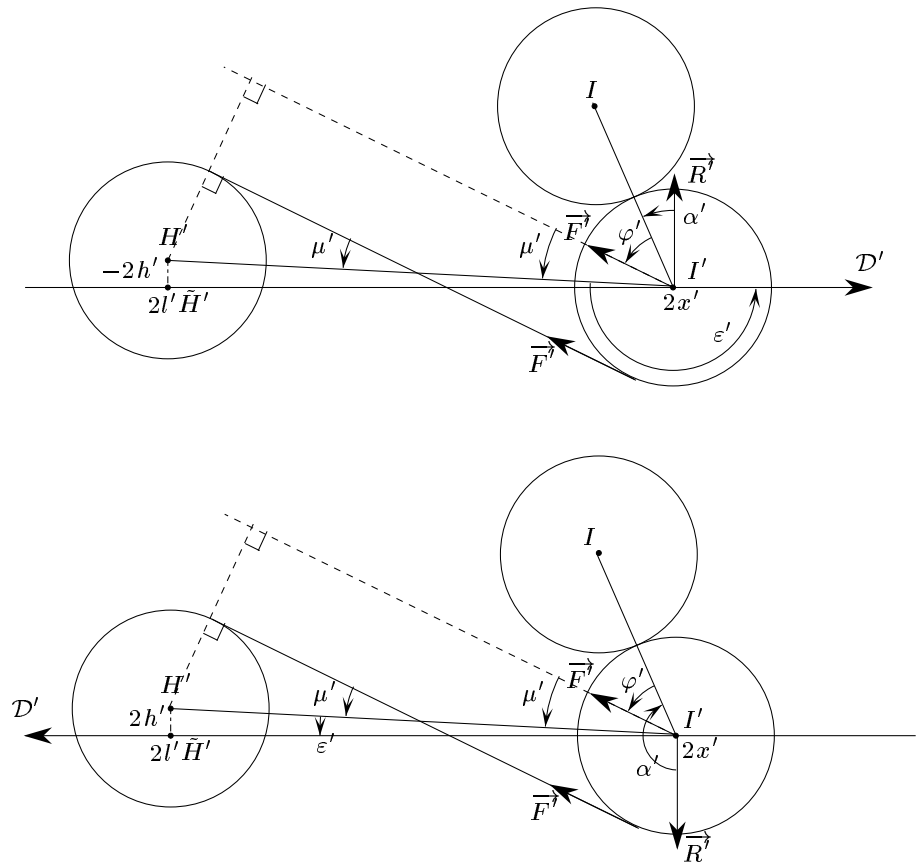

Fig. 14. For computing Eq. (8).

Lemma 13. There is a finite number of distinct values of $\alpha$ in the set of solutions of System (2) in the variables $\left(\alpha, \alpha+\varphi, \alpha^{\prime}+\varphi^{\prime}\right) \in\left(S^{1}\right)^{3}$.

Proof. Using the variable substitution $x=\tan \left(\frac{\alpha}{2}\right), y=\tan \left(\frac{\alpha+\varphi}{2}\right)$ and $z=$ $\tan \left(\frac{\alpha^{\prime}+\varphi^{\prime}}{2}\right)$, we transform System (2) into an algebraic system where $x, y, z$ are the indeterminates and $h, l, h^{\prime}, l^{\prime}, \sin \gamma, \cos \gamma$ are six independent parameters. Let $E_{i}=0(i \in\{1,2,3\})$ denote the algebraic equation obtained from the $i$-th equation of System (2).

Using MAPLE, we compute the resultant $Q(x, z)$ of $E_{1}$ and $E_{2}$ with respect to the indeterminate $y .^{3}$ Then, we compute the resultant $R(x)$ of $Q(x, z)$ and $E_{3}$ with respect to the indeterminate $z \cdot R(x)$ is a univariate polynomial of degree 24 . Any root of System (2) verifies $R(\tan (\alpha / 2))=0 .{ }^{3}$ Therefore, if $R(x) \not \equiv 0$, there is a finite number of distinct values of $\alpha$ in the set of solutions of System of equations (2) in the variables $\left(\alpha, \alpha+\varphi, \alpha^{\prime}+\varphi^{\prime}\right) \in\left(S^{1}\right)^{3}$.

We now prove that $R(x) \not \equiv 0$. The leading and the trailing monomials of $R(x)$ are

$$
\begin{gathered}
2^{8}(h+\delta)^{4} \sin ^{8} \gamma\left(\left(1+l^{\prime} \sin \gamma\right)^{2}+h^{\prime 2} \sin ^{2} \gamma\right)^{2} x^{24} \\
2^{8}(h-\delta)^{4} \sin ^{8} \gamma\left(\left(1-l^{\prime} \sin \gamma\right)^{2}+h^{\prime 2} \sin ^{2} \gamma\right)^{2}
\end{gathered}
$$


Both coefficients are zero only if $h=\delta=0$ or if $\left(h, h^{\prime}, l^{\prime}\right)$ is equal to either $(\delta, 0,-1 / \sin \gamma)$ or $(-\delta, 0,1 / \sin \gamma)$ since $\sin \gamma \neq 0$, by hypothesis. If $h=\delta=0$ then $\cos (\alpha+\varphi)=0$ and so $E_{2}=0$; then, the leading and the trailing coefficients (with respect to $x$ ) of the resultant of $E_{1}$ and $E_{3}$ with respect to $z$ are

$$
\begin{aligned}
& 4 \sin ^{2} \gamma(y-1)^{4}\left(\left(1+l^{\prime} \sin \gamma\right)^{2}+h^{\prime 2} \sin ^{2} \gamma\right), \\
& 4 \sin ^{2} \gamma(y+1)^{4}\left(\left(1-l^{\prime} \sin \gamma\right)^{2}+h^{\prime 2} \sin ^{2} \gamma\right),
\end{aligned}
$$

where $y=\sin (\alpha+\varphi)= \pm 1$. These two coefficients are zero only if $\left(h^{\prime}, l^{\prime}\right)=$ $(0, \pm 1 / \sin \gamma)$. It thus follows from $h=\delta=0$ that $\left(h, h^{\prime}, l^{\prime}\right)$ is equal to $(\delta, 0,-1 / \sin \gamma)$ or to $(-\delta, 0,1 / \sin \gamma)$.

Hence, in both cases, the leading and the trailing coefficients of $R(x)$ are zero only if $\left(h, h^{\prime}, l^{\prime}\right)$ is equal to $(\delta, 0,-1 / \sin \gamma)$ or to $(-\delta, 0,1 / \sin \gamma)$. We substitute, in turn, $\left(h, h^{\prime}, l^{\prime}\right)$ for those values in $R(x)$. When $\left(h, h^{\prime}, l^{\prime}\right)=(\delta, 0,-1 / \sin \gamma)$, the new leading and trailing monomials of $R(x)$ are

$$
\begin{gathered}
2^{12} \delta^{4} \sin ^{4} \gamma\left((l \sin \gamma+\cos \gamma)^{2}+\delta^{2} \sin ^{2} \gamma\right)^{2} x^{20}, \\
2^{14} \sin ^{4} \gamma\left(\delta^{2}-4\right)(l \sin \gamma-\cos \gamma)^{4} x^{2},
\end{gathered}
$$

and when $\left(h, h^{\prime}, l^{\prime}\right)=(-\delta, 0,1 / \sin \gamma)$, the new leading and trailing monomials of $R(x)$ are

$$
\begin{gathered}
2^{14} \sin ^{4} \gamma\left(\delta^{\prime 2}-4\right)(l \sin \gamma+\cos \gamma)^{4} x^{22}, \\
2^{12} \delta^{\prime 4} \sin ^{4} \gamma\left((l \sin \gamma-\cos \gamma)^{2}+\delta^{2} \sin ^{2} \gamma\right)^{2} x^{4} .
\end{gathered}
$$

We study the two cases $\delta^{\prime}=0$ and $\delta^{\prime}=1$. If $\delta^{\prime}=0, R(x) \equiv 0$ only if $\left(h, h^{\prime}, l^{\prime}, l\right)$ is equal to either $(\delta, 0,-1 / \sin \gamma, \cot \gamma)$ or $(-\delta, 0,1 / \sin \gamma,-\cot \gamma)$. For those values of $\left(h, h^{\prime}, l^{\prime}, l\right)$ the leading or the trailing coefficient of the new resultant $R(x)$ is $2^{16} \delta^{4} \sin ^{8} \gamma$. Furthermore, if $\delta=0$, the leading or the trailing coefficient of the new resultant $R(x)$ is $-2^{20} \sin ^{8} \gamma$, which is not zero. If $\delta^{\prime}=1, R(x) \equiv 0$ only if $\delta=0$, $\gamma= \pm \pi / 2$ and $\left(h, h^{\prime}, l^{\prime}, l\right)=(0,0, \pm 1,0)$. Then, as before $E_{2}=0$, and the resultant of $E_{1}$ and $E_{3}$ with respect to $z$ is a univariate polynomial in $x$ whose coefficient of degree 2 if $l^{\prime}=1$ or coefficient of degree 4 if $l^{\prime}=-1$ is equal to 64 , which is not zero.

Hence, the univariate polynomial in $x, R(x)$ or, if $E_{2}=0$, the resultant of $E_{1}$ and $E_{3}$ with respect to $z$, is not identically zero. This concludes the proof since $x=\tan \left(\frac{\alpha}{2}\right)$.

Lemma 14. A value $\alpha$ determines $O(1)$ paths $\mathcal{P}$.

Proof. $\alpha$ determines uniquely $\alpha^{\prime}$, by Eq. (3), and thus the position of $\mathcal{C}$ and $\mathcal{C}^{\prime}$, by Eq. (4). Path $\mathcal{P}$ is then characterized by the orientation of its four circular arcs. 


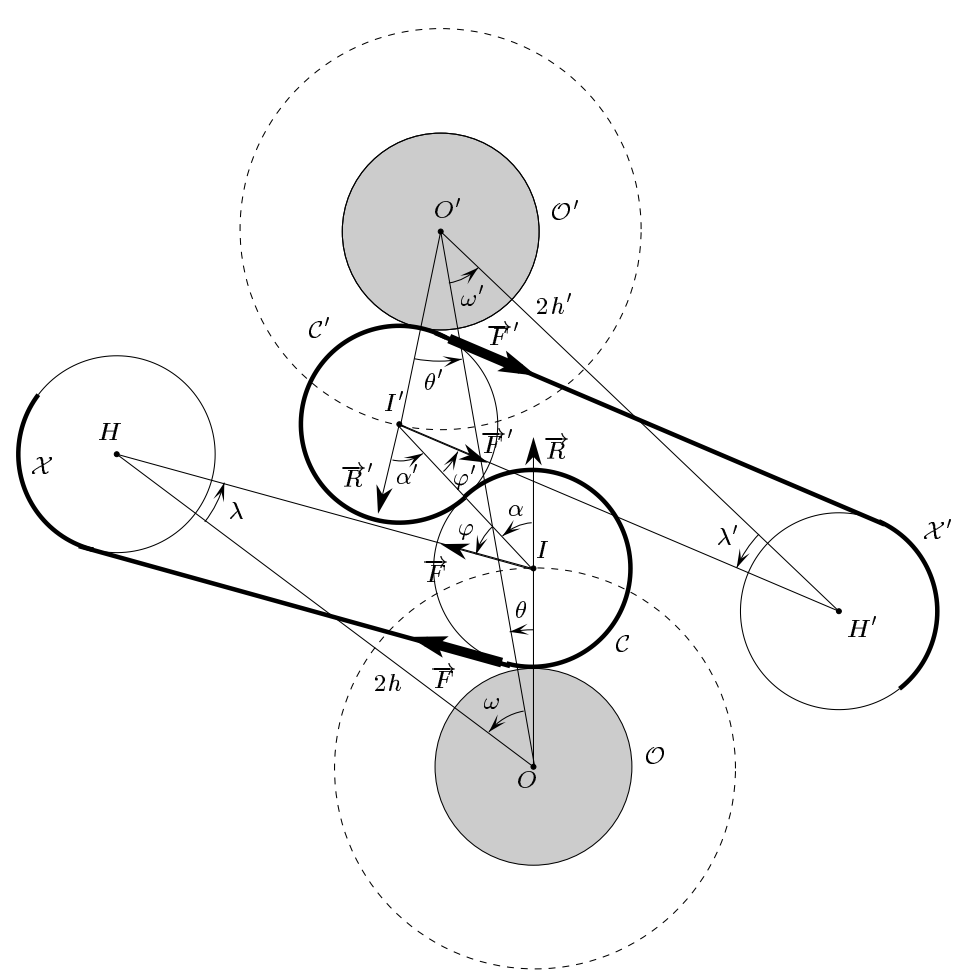

Fig. 15. Mechanical device where both obstacle edges are circular $(\mathcal{P}$ has the same orientation on $\mathcal{X}$ and $\mathcal{C}$, and on $\mathcal{X}^{\prime}$ and $\mathcal{C}^{\prime}$ ).

\section{Case 2: Both obstacle edges are circular arcs}

The proof is similar to the previous case. We compute a system of 4 equations in 4 indeterminates whose solutions correspond to potential equilibriums of the mechanical device. Then, we consider a univariate polynomial $R(x)$ by cascading resultants as explained above. However, in this case, computing such a polynomial $R(x)$ exceeds the capabilities of the current computer algebra systems. We thus only compute the leading monomial of $R(x)$ and show that it is not identically equal to zero, which implies that $R(x) \not \equiv 0$.

Let $O$ and $O^{\prime}$ be the centers of the circles (of unit radius) supporting $\mathcal{O}$ and $\mathcal{O}^{\prime}$, respectively. Let $2 d$ be the length of $O O^{\prime}$. Let $2 h$ and $2 h^{\prime}$ be the lengths of $O H$ and $O^{\prime} H^{\prime}$ respectively. Let $\omega=\angle\left(\overrightarrow{O O^{\prime}}, \overrightarrow{O H}\right)$ and $\omega^{\prime}=\angle\left(\overrightarrow{O^{\prime} O}, \overrightarrow{O^{\prime} H^{\prime}}\right)$ (see Figure 15).

First notice that if $d$ is equal to 0 or 1 then $\mathcal{P}$ is not optimal since it can be shortened by a Dubins' perturbation (see Figure 16 (a) and (b)). Similarly, if $h=0$ then either $\mathcal{P}$ is of type $X S \bar{C} \bar{C}^{\prime} S X^{\prime}$ where $X$ lies on $\mathcal{O}$ and then it is not optimal since it can be shortened by the same Dubins' perturbation (see Figure 16 (c)), or it is of type $X \bar{C} \bar{C}^{\prime} S X^{\prime}$ and then it is not optimal by hypothesis (since one $S$-segment 
is reduced to a point). Thus we can assume that $d$ is not equal to 0 or 1 and $h$ is not equal to 0 (and similarly for $h^{\prime}$ ).

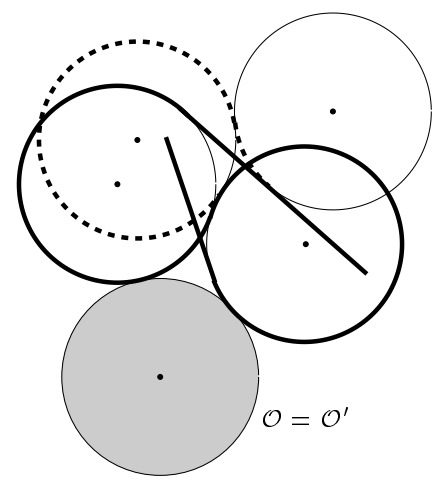

$(a): d=0$

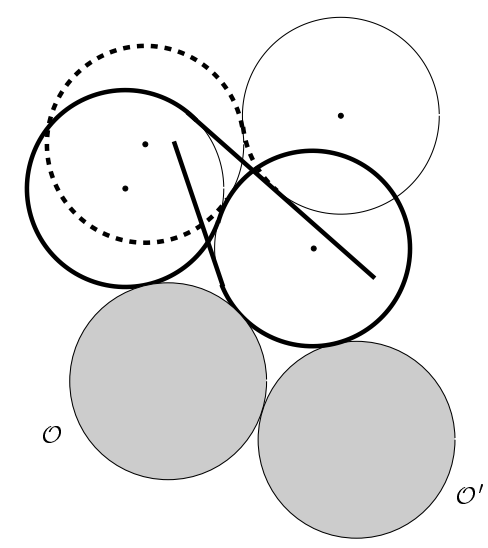

(b) $: d=1$

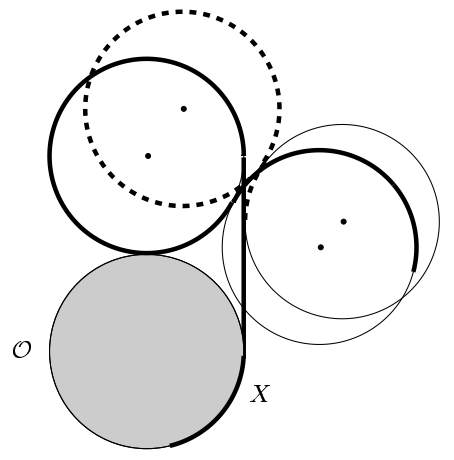

$(c): h=0$

Fig. 16. Dubins' length-reducing perturbation for path $\mathcal{P}$ if $d=0$ or 1 or if $h=0$.

Lemma 15. Path $\mathcal{P}$ is optimal only if

$$
\left\{\begin{array}{l}
\sin \left(\alpha^{\prime}-\alpha\right)+\sin \alpha^{\prime} \sin (\alpha+\varphi)-\sin \alpha \sin \left(\alpha^{\prime}+\varphi^{\prime}\right)=0 \\
2 \cos \alpha+2 \cos \alpha^{\prime}+2 \cos \left(\alpha^{\prime}-\alpha\right)+3-d^{2}=0 \\
h \sin (\varphi-\omega)+h \sin \left(\alpha^{\prime}+\varphi-\omega\right)+h \sin (\alpha+\varphi-\omega)-d \sin (\alpha+\varphi)+\delta d=0 \\
h^{\prime} \sin \left(\varphi^{\prime}-\omega^{\prime}\right)+h^{\prime} \sin \left(\alpha+\varphi^{\prime}-\omega^{\prime}\right)+h^{\prime} \sin \left(\alpha^{\prime}+\varphi^{\prime}-\omega^{\prime}\right)-d \sin \left(\alpha^{\prime}+\varphi^{\prime}\right)+\delta^{\prime} d=0
\end{array}\right.
$$

where $\delta$ (resp. $\left.\delta^{\prime}\right)$ is zero if path $\mathcal{P}$ has the same orientation on $\mathcal{X}$ and $\mathcal{C}$ (resp. $\mathcal{X}^{\prime}$ and $\left.\mathcal{C}^{\prime}\right)$ and 1 otherwise.

Proof. The first equation of System (9) is given by Lemma 11. Now, let $\theta=$ $\angle\left(\overrightarrow{O I}, \overrightarrow{O O^{\prime}}\right)$ and $\theta^{\prime}=\angle\left(\overrightarrow{O^{\prime} I^{\prime}}, \overrightarrow{O^{\prime} O}\right)$ (see Figure 15). Considering the polygon $\left(O I I^{\prime} O^{\prime}\right)$ in Figure 15, we get

$$
\begin{aligned}
& \alpha^{\prime}-\theta^{\prime}=\alpha-\theta[2 \pi], \\
& \cos \theta+\cos (\alpha-\theta)+\cos \theta^{\prime}=d, \\
& \sin \theta-\sin (\alpha-\theta)+\sin \theta^{\prime}=0 .
\end{aligned}
$$

Considering in turn $\left((E q .11)^{2}+(E q .12)^{2}\right),((E q .11) \sin \theta-(E q .12) \cos \theta)$, $((E q .11) \cos \theta+(E q .12) \sin \theta),\left((E q .11) \sin \theta^{\prime}-(E q .12) \cos \theta^{\prime}\right)$ and $\left((E q .11) \cos \theta^{\prime}+\right.$ (Eq.12) $\left.\sin \theta^{\prime}\right)$, we obtain

$$
2 \cos \alpha+2 \cos \alpha^{\prime}+2 \cos \left(\alpha^{\prime}-\alpha\right)+3-d^{2}=0,
$$




$$
\begin{aligned}
& d \sin \theta=\sin \alpha-\sin \left(\alpha^{\prime}-\alpha\right), \\
& d \cos \theta=1+\cos \alpha+\cos \left(\alpha^{\prime}-\alpha\right), \\
& d \sin \theta^{\prime}=\sin \alpha^{\prime}+\sin \left(\alpha^{\prime}-\alpha\right), \\
& d \cos \theta^{\prime}=1+\cos \alpha^{\prime}+\cos \left(\alpha^{\prime}-\alpha\right) .
\end{aligned}
$$

Eq. (13) is the second equation of System (9). We now show how to compute the two other equations of System (9) for the possible orientations of $X$ and $X^{\prime}$.

- $\mathcal{P}$ has the same orientation on $\mathcal{X}$ and $\mathcal{C}$ (see Figure 15).

Let $\lambda=\angle(\overrightarrow{H O}, \overrightarrow{H I})$. Considering the triangle $(O H I)$ yields

$$
\frac{\sin (\alpha+\varphi)}{2 h}=\frac{\sin \lambda}{2} \text {. }
$$

Since $\lambda=\alpha+\varphi-\theta-\omega[2 \pi]$, we get

$$
h \sin (\alpha+\varphi-\theta-\omega)-\sin (\alpha+\varphi)=0 .
$$

We expand the equation with respect to $\theta$, and simplify it using Eqs. (14) and (15):

$$
h \sin (\varphi-\omega)+h \sin \left(\alpha^{\prime}+\varphi-\omega\right)+h \sin (\alpha+\varphi-\omega)-d \sin (\alpha+\varphi)=0 .
$$

This equation is the third equation of System (9) since $\delta$ is here equal to zero.

- $\mathcal{P}$ has the same orientation on $\mathcal{X}^{\prime}$ and $\mathcal{C}^{\prime}$.

Similarly as above, we get, considering the triangle $\left(O^{\prime} H^{\prime} I^{\prime}\right)$,

$$
\frac{\sin \left(\alpha^{\prime}+\varphi^{\prime}\right)}{2 h^{\prime}}=\frac{\sin \lambda^{\prime}}{2}
$$

where $\lambda^{\prime}=\alpha^{\prime}+\varphi^{\prime}-\theta^{\prime}-\omega^{\prime}[2 \pi]$. It follows

$$
h^{\prime} \sin \left(\alpha^{\prime}+\varphi^{\prime}-\theta^{\prime}-\omega^{\prime}\right)-\sin \left(\alpha^{\prime}+\varphi^{\prime}\right)=0
$$

which we expand and simplify using Eqs. (16) and (17). We obtain

$$
h^{\prime} \sin \left(\varphi^{\prime}-\omega^{\prime}\right)+h^{\prime} \sin \left(\alpha+\varphi^{\prime}-\omega^{\prime}\right)+h^{\prime} \sin \left(\alpha^{\prime}+\varphi^{\prime}-\omega^{\prime}\right)-d \sin \left(\alpha^{\prime}+\varphi^{\prime}\right)=0
$$

which is the last equation of System (9) since $\delta$ is here equal to zero.

- $\mathcal{P}$ has opposite orientations on $\mathcal{X}$ and $\mathcal{C}$ (see Figure 17).

Let $\mu=\angle(\vec{F}, \overrightarrow{I H})$. Considering the triangle $(O H I)$ yields

$$
\frac{\sin (\alpha+\varphi+\mu)}{2 h}=\frac{\sin \lambda}{2}=\frac{\sin (\theta+\omega)}{I H} .
$$

Since $\lambda=\alpha+\varphi+\mu-\theta-\omega[2 \pi]$, we get

$$
\begin{aligned}
2 \sin (\theta+\omega) & =I H \sin (\alpha+\varphi-\theta-\omega) \cos \mu+I H \cos (\alpha+\varphi-\theta-\omega) \sin \mu, \\
2 h \sin (\theta+\omega) & =I H \sin (\alpha+\varphi) \cos \mu+I H \cos (\alpha+\varphi) \sin \mu .
\end{aligned}
$$

Eliminating $\cos \mu$ from these two equations gives

$$
\begin{aligned}
& 2 \sin (\theta+\omega) \sin (\alpha+\varphi)-2 h \sin (\theta+\omega) \sin (\alpha+\varphi-\theta-\omega)= \\
& I H \cos (\alpha+\varphi-\theta-\omega) \sin \mu \sin (\alpha+\varphi)-I H \cos (\alpha+\varphi) \sin \mu \sin (\alpha+\varphi-\theta-\omega) .
\end{aligned}
$$




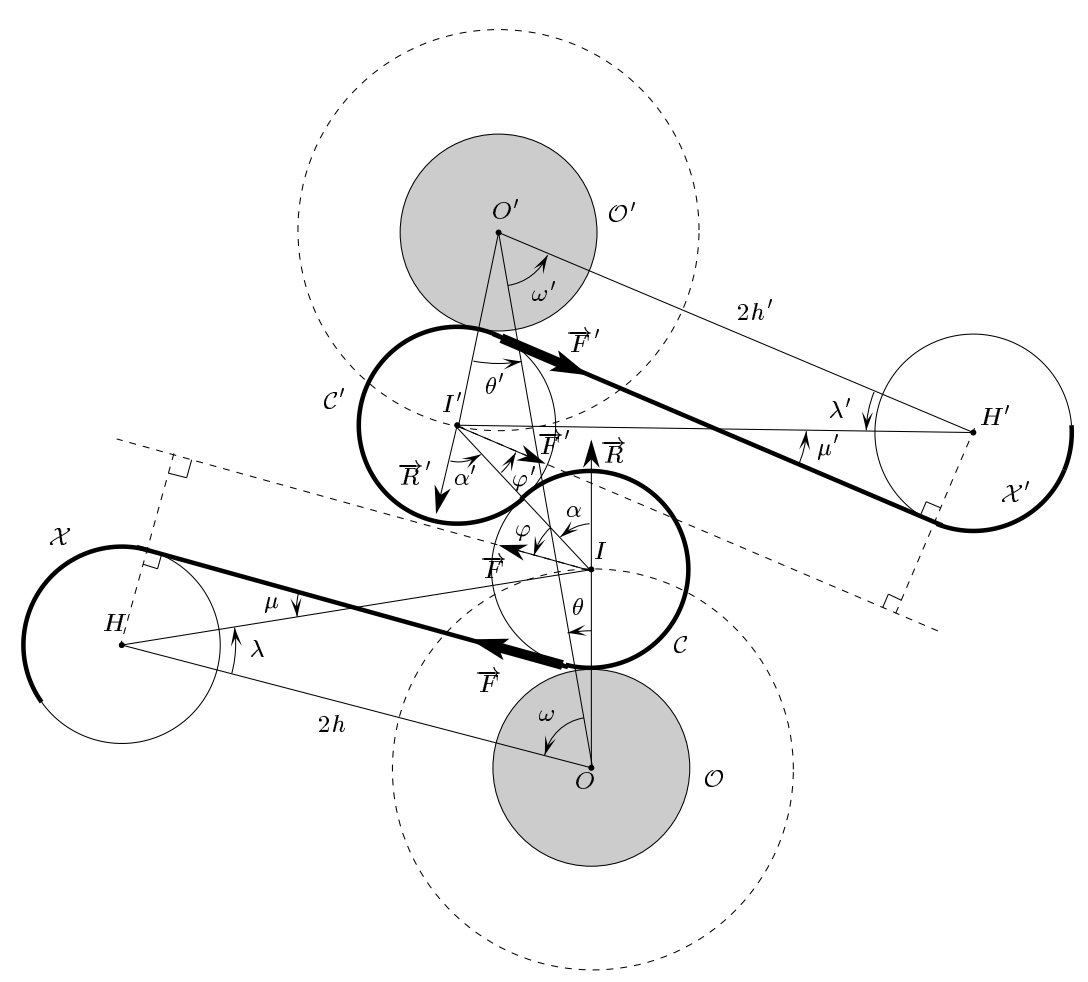

Fig. 17. For the proof of Lemma $15\left(\mathcal{P}\right.$ has opposite orientation on $\mathcal{X}$ and $\mathcal{C}$, and on $\mathcal{X}^{\prime}$ and $\left.\mathcal{C}^{\prime}\right)$.

We simplify this equation into

$$
2 \sin (\theta+\omega) \sin (\alpha+\varphi)-2 h \sin (\theta+\omega) \sin (\alpha+\varphi-\theta-\omega)=I H \sin \mu \sin (\theta+\omega) .
$$

As $\sin \mu=2 / I H$ (see Figure 17), we have

$$
\sin (\theta+\omega)(h \sin (\alpha+\varphi-\theta-\omega)-\sin (\alpha+\varphi)+1)=0 .
$$

We can assume that $\sin (\theta+\omega) \neq 0$ because otherwise $I$ lies on the straight line $\mathrm{OH}$ and the number of such paths is less than four. Thus, the mobile object is at an equilibrium only if

$$
h \sin (\alpha+\varphi-\theta-\omega)-\sin (\alpha+\varphi)+1=0 .
$$

Similarly as above, we expand that equation with respect to $\theta$ and simplify it, thanks to Eqs. (14) and (15). We get

$$
\sin (\varphi-\omega)+h \sin \left(\alpha^{\prime}+\varphi-\omega\right)+h \sin (\alpha+\varphi-\omega)-d \sin (\alpha+\varphi)+d=0
$$

which is the third equation of System (9) since $\delta$ is here equal to 1 .

- $\mathcal{P}$ has opposite orientations on $\mathcal{X}^{\prime}$ and $\mathcal{C}^{\prime}$.

Similarly as above, we obtain

$$
h^{\prime} \sin \left(\alpha^{\prime}+\varphi^{\prime}-\theta^{\prime}-\omega^{\prime}\right)-\sin \left(\alpha^{\prime}+\varphi^{\prime}\right)+1=0
$$


which we simplify using Eqs. (16) and (17), and gives

$$
h^{\prime} \sin \left(\varphi^{\prime}-\omega^{\prime}\right)+h^{\prime} \sin \left(\alpha+\varphi^{\prime}-\omega^{\prime}\right)+h^{\prime} \sin \left(\alpha^{\prime}+\varphi^{\prime}-\omega^{\prime}\right)
$$

which is the last equation of System (9) since $\delta$ is here equal to 1 .

Lemma 16. There is a finite number of distinct values of $\left(\alpha, \alpha^{\prime}\right)$ in the set of solutions of System (9) in the variables $\left(\alpha, \alpha^{\prime}, \varphi, \varphi^{\prime}\right) \in\left(S^{1}\right)^{4}$.

Proof. We expand each equation of System (9) and apply the variable substitution $x=\tan (\alpha / 2), y=\tan \left(\alpha^{\prime} / 2\right), z=\tan (\varphi / 2)$ and $t=\tan \left(\varphi^{\prime} / 2\right)$. This yields an algebraic system consisting of four equations where $x, y, z, t$ are the four indeterminates and $\omega, \omega^{\prime}, d, h, h^{\prime}, l^{\prime}$ are six independent parameters. Let $E_{i}=0(i \in\{1, \ldots, 4\})$ denote the algebraic equation obtained from the $i$-th equation of System (9). We compute $^{\mathrm{a}}$ the resultant $E_{14}$ of $E_{1}$ and $E_{4}$ with respect to the indeterminate $t^{3} E_{14}$ can be written as $16\left(1+y^{2}\right) E_{14}^{\prime}$. We compute ${ }^{\mathrm{b}}$ the resultant $Q$ of $E_{14}^{\prime}$ and $E_{2}$ with respect to the indeterminate $y$. We also compute the resultant $T$ of $E_{2}$ and $E_{3}$ with respect to the indeterminate $y . Q$ and $T$ are two polynomials where $x$ and $z$ are the indeterminates.

Now, let $R$ be the resultant of $Q$ and $T$ with respect to $z$. $R$ is a univariate polynomial in the indeterminate $x$ and we want to show that $R(x) \not \equiv 0$. Let

$$
\begin{aligned}
& Q=q_{0}+q_{1} z+\ldots+q_{n} z^{n}, q_{n} \neq 0, \\
& T=t_{0}+t_{1} z+\ldots+t_{m} z^{m}, t_{m} \neq 0,
\end{aligned}
$$

where the $q_{i}$ and the $t_{i}$ are univariate polynomials in the variable $x$. The resultant $R$ of $Q$ and $T$ with respect to $z$ is the determinant of the $(n+m) \times(n+m)$ Sylvester matrix of $Q$ and $T$ with respect to $z:^{3}$

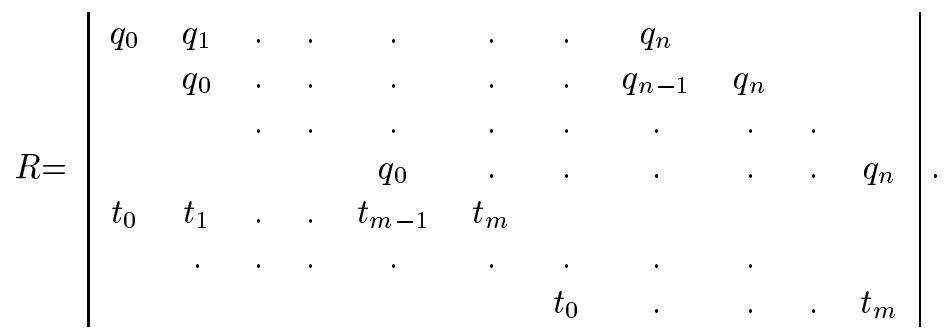

The resultant $R(x)$ is too big to be computed with existing computer algebra systems but we are able to compute its leading monomial. The polynomials $q_{i}$ appear in the first $m$ rows of the Sylvester determinant and the polynomials $t_{i}$ appear in the last $n$ rows. Thus, the degree of $R$ with respect to $x$ is at most

$$
m \max _{i \in\{0, \ldots, n\}} \operatorname{degree}\left(q_{i}(x)\right)+n \max _{i \in\{0, \ldots, m\}} \operatorname{degree}\left(t_{i}(x)\right)=168 .
$$

${ }^{a}$ We used AXIOM on a Sun Sparc-10 $4 \times 72 \mathrm{Mhz}$ with $512 \mathrm{MB}$ of main memory. Notice that several polynomials considered here are two big to be computed with MAPLE.

${ }^{\mathrm{b}}$ This computation takes roughly eleven hours and the process exceeds 130MB. 
Since existing computer algebra systems cannot compute the resultant $R$, we replace in the Sylvester determinant each $q_{i}(x)$ by its monomial of highest degree if degree $\left(q_{i}(x)\right)=\max _{i \in\{0, \ldots, n\}} \operatorname{degree}\left(q_{i}(x)\right)$ and by 0 otherwise, and each $t_{i}(x)$ by its monomial of highest degree if $\operatorname{degree}\left(t_{i}(x)\right)=\max _{i \in\{0, \ldots, m\}}$

degree $\left(t_{i}(x)\right)$ and by 0 otherwise. We then compute the determinant and obtain

$2^{56} d^{8}\left(d^{2}-1\right)^{32} h^{16}\left(h^{\prime 2} \sin ^{2} \omega^{\prime}+\left(h^{\prime} \cos \omega^{\prime}-d\right)^{2}\right)^{4}\left(d^{2} h^{\prime 2} \sin ^{2} \omega^{\prime}+\left(d h^{\prime} \cos \omega^{\prime}-1\right)^{2}\right)^{4} x^{168}$.

When this monomial is not zero, its degree is 168 and thus it is the leading monomial of $R(x)$ because the degree of $R(x)$ is at most 168 . Thus, $R(x) \equiv 0$ only if this monomial is zero. Since $d$ is not equal to 0 or 1 and $h \neq 0$, this monomial is zero only if $\left(\omega^{\prime}, h^{\prime}\right)$ is equal to $(0, d),(\pi,-d),(0,1 / d)$, or $(\pi,-1 / d)$. Moreover, since $h^{\prime}$ and $d$ are both distances, $\left(\omega^{\prime}, h^{\prime}\right)$ can only be equal to $(0, d)$ or $(0,1 / d)$. We replace $\omega^{\prime}$ by 0 and $h^{\prime}$ by $d$ or $1 / d$ in $Q$ and $T$. We then apply the same procedure as above and show that, in both cases, the new leading monomial of $R(x)$ is

$$
2^{72} d^{16}\left(d^{2}-1\right)^{32} h^{16} x^{152} .
$$

Since $d$ is not equal to 0 or $1, R(x) \not \equiv 0$. Therefore, there is a finite number of distinct values of $\alpha=2 \arctan x$ in the set of solutions of System (9) in the variables $\left(\alpha, \alpha^{\prime}, \varphi, \varphi^{\prime}\right) \in\left(S^{1}\right)^{4}$.

The second equation of System (9) gives with the variable substitution $y=$ $\tan \left(\alpha^{\prime} / 2\right)$ that

$$
2 \cos \alpha+2 \frac{1-y^{2}}{1+y^{2}}+2 \cos \alpha \frac{1-y^{2}}{1+y^{2}}+2 \sin \alpha \frac{2 y}{1+y^{2}}+3-d^{2}=0
$$

or equivalently

$$
\left(1-d^{2}\right) y^{2}+4 \sin \alpha y+4 \cos \alpha+5-d^{2}=0 .
$$

This polynomial is not identically equal to zero since $d$ is a non-negative distance distinct from 1 . Thus $\alpha$ determines at most two $\alpha^{\prime}=2 \arctan y$. It follows that there is a finite number of distinct values of $\alpha$ and $\alpha^{\prime}$ in the set of solutions of System (2) in the variables $\left(\alpha, \alpha^{\prime}, \varphi, \varphi^{\prime}\right) \in\left(S^{1}\right)^{4}$.

Lemma 17. A couple of values $\left(\alpha, \alpha^{\prime}\right)$ determines $O(1)$ paths $\mathcal{P}$.

Proof. By Eqs. (14), (15), (16) and (17), $\alpha$ and $\alpha^{\prime}$ define uniquely $\theta$ and $\theta^{\prime}$ which characterize the position of $\mathcal{C}$ and $\mathcal{C}^{\prime}$. Path $\mathcal{P}$ is then characterized by the orientation of its four circular arcs.

\section{Case 3: One obstacle edge is a line segment and the other is a circular arc}

The proof is similar to the previous case. We assume, without loss of generality, that $\mathcal{O}$ and $\mathcal{O}^{\prime}$ are respectively a circular arc and a line segment. 


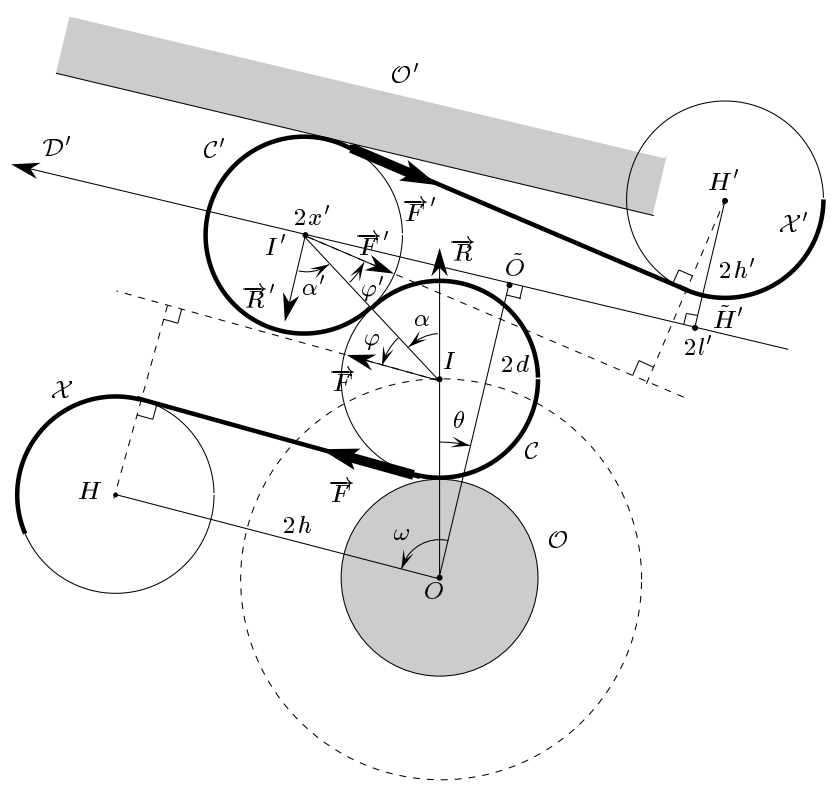

Fig. 18. Mechanical device where one obstacle edge is a line segment and the other is circular.

Let $\mathcal{D}^{\prime}$ be the line parallel to $\mathcal{O}^{\prime}$ passing through $I^{\prime}$ and oriented such that $\angle\left(\overrightarrow{R^{\prime}}, \mathcal{D}^{\prime}\right)=-\pi / 2$ (see Figure 18). Let $O$ be the center of the circle (of unit radius) supporting $\mathcal{O}$. Let $\tilde{O}$ be the orthogonal projection of $O$ onto $\mathcal{D}^{\prime}$ and $2 d$ the length of $O \tilde{O}$. Let $2 h$ be the length of $O H$. Let $\tilde{H}^{\prime}$ be the orthogonal projection of $H^{\prime}$ onto $\mathcal{D}^{\prime}$. Let $2 x^{\prime}$ and $2 l^{\prime}$ be the algebraic lengths of $\tilde{O} I^{\prime}$ and $\tilde{O} \tilde{H}^{\prime}$ on the oriented line $\mathcal{D}^{\prime}$. Let $2 h^{\prime}$ be the algebraic distance between $H^{\prime}$ and $\mathcal{D}^{\prime}$ with $2 h^{\prime}>0$ if and only if $\overrightarrow{\tilde{H}^{\prime} H^{\prime}}$ and $\overrightarrow{R^{\prime}}$ have opposite orientations. Let $\omega=\angle(\overrightarrow{O O}, \overrightarrow{O H})$.

Notice that if $d=0$ then $\mathcal{P}$ is not optimal since it can be shortened by a Dubins' perturbation (see Figure 19). Also, as in the previous case, if $h=0$ then either $\mathcal{P}$ is of type $X S \bar{C} \bar{C}^{\prime} S X^{\prime}$ where $X$ lies on $\mathcal{O}$ and then it is not optimal since it can be shortened by a Dubins' perturbation (see Figure 16 (c)), or it is of type $X \bar{C} \bar{C}^{\prime} S X^{\prime}$ and then it is not optimal by hypothesis. Thus we can assume that $d$ and $h$ are not equal to 0 .

Lemma 18. Path $\mathcal{P}$ is optimal only if

$$
\left\{\begin{array}{l}
\sin \left(\alpha^{\prime}-\alpha\right)+\sin \alpha^{\prime} \sin (\alpha+\varphi)-\sin \alpha \sin \left(\alpha^{\prime}+\varphi^{\prime}\right)=0 \\
\cos \left(\alpha^{\prime}-\alpha\right)+\cos \alpha^{\prime}=d \\
h \sin \left(\alpha^{\prime}+\varphi-\omega\right)-\sin (\alpha+\varphi)+\delta=0 \\
h^{\prime} \sin \left(\alpha^{\prime}+\varphi^{\prime}\right)+\cos \left(\alpha^{\prime}+\varphi^{\prime}\right)\left(\sin \alpha^{\prime}+\sin \left(\alpha^{\prime}-\alpha\right)-l^{\prime}\right)+\delta^{\prime}=0
\end{array}\right.
$$

where $\delta$ (resp. $\left.\delta^{\prime}\right)$ is zero if path $\mathcal{P}$ has the same orientation on $\mathcal{X}$ and $\mathcal{C}$ (resp. $\mathcal{X}^{\prime}$ and $\mathcal{C}^{\prime}$ ) and 1 otherwise. 

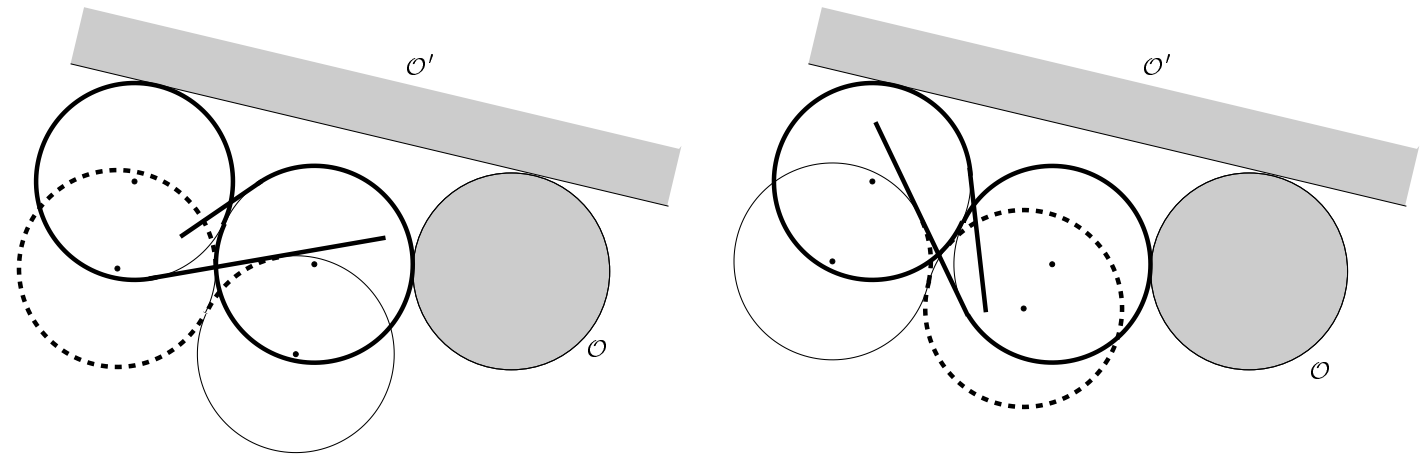

Fig. 19. Dubins' length-reducing perturbation for path $\mathcal{P}$ if $d=0$.

Proof. The first equation of System (20) is given by Lemma 11. Now, let $\theta=$ $\angle(\overrightarrow{O I}, \overrightarrow{O O})$. Considering the polygon $\left(O I I^{\prime} \tilde{O}\right)$ in Figure 18 , we get

$$
\begin{aligned}
& \alpha^{\prime}=\alpha-\theta[2 \pi], \\
& \cos \theta+\cos (\alpha-\theta)=d, \\
& -\sin \theta+\sin (\alpha-\theta)=x^{\prime} .
\end{aligned}
$$

It follows

$$
\begin{aligned}
\cos \left(\alpha^{\prime}-\alpha\right)+\cos \alpha^{\prime} & =d, \\
\sin \left(\alpha^{\prime}-\alpha\right)+\sin \alpha^{\prime} & =x^{\prime} .
\end{aligned}
$$

Eq. (22) is the second equation of System (20). We now show how to compute the two other equations of System (20) for each possible orientation of $X$ and $X^{\prime}$.

- If $\mathcal{P}$ has the same orientation on $\mathcal{X}$ and $\mathcal{C}$, then Eq. (18) still holds:

$$
h \sin (\alpha+\varphi-\theta-\omega)-\sin (\alpha+\varphi)=0 .
$$

Thus, since $\alpha^{\prime}=\alpha-\theta[2 \pi]$,

$$
h \sin \left(\alpha^{\prime}+\varphi-\omega\right)-\sin (\alpha+\varphi)=0
$$

which is the third equation of System (20).

- If $\mathcal{P}$ has opposite orientations on $\mathcal{X}$ and $\mathcal{C}$, then Eq. (19) still holds:

$$
h \sin (\alpha+\varphi-\theta-\omega)-\sin (\alpha+\varphi)+1=0 .
$$

Since $\alpha^{\prime}=\alpha-\theta[2 \pi]$, we get

$$
h \sin \left(\alpha^{\prime}+\varphi-\omega\right)-\sin (\alpha+\varphi)+1=0
$$


which is the third equation of System (20).

- If $\mathcal{P}$ has the same orientation on $\mathcal{X}^{\prime}$ and $\mathcal{C}^{\prime}$, then Eq. (5) still holds:

$$
\tan \left(\alpha^{\prime}+\varphi^{\prime}\right)=\frac{l^{\prime}-x^{\prime}}{h^{\prime}} .
$$

By Eq. (23), we obtain

$$
h^{\prime} \sin \left(\alpha^{\prime}+\varphi^{\prime}\right)+\cos \left(\alpha^{\prime}+\varphi^{\prime}\right)\left(\sin \alpha^{\prime}+\sin \left(\alpha^{\prime}-\alpha\right)-l^{\prime}\right)=0
$$

which is the fourth equation of System (20).

- If $\mathcal{P}$ has opposite orientations on $\mathcal{X}^{\prime}$ and $\mathcal{C}^{\prime}$, then Eq. (7) still holds:

$$
\left(x^{\prime}-l^{\prime}\right) \cos \left(\alpha^{\prime}+\varphi^{\prime}\right)+h^{\prime} \sin \left(\alpha^{\prime}+\varphi^{\prime}\right)=-1 .
$$

Using Eq. (23), we get

$$
h^{\prime} \sin \left(\alpha^{\prime}+\varphi^{\prime}\right)+\cos \left(\alpha^{\prime}+\varphi^{\prime}\right)\left(\sin \alpha^{\prime}+\sin \left(\alpha^{\prime}-\alpha\right)-l^{\prime}\right)+1=0
$$

which is the fourth equation of System (20).

Lemma 19. There is a finite number of distinct values of $\left(\alpha, \alpha^{\prime}\right)$ in the set of solutions of System (20) in the variables $\left(\alpha, \alpha^{\prime}, \varphi, \varphi^{\prime}\right) \in\left(S^{1}\right)^{4}$.

Proof. We consider the variable substitution $x=\tan (\alpha / 2), y=\tan \left(\alpha^{\prime} / 2\right), z=$ $\tan (\varphi / 2)$ and $t=\tan \left(\varphi^{\prime} / 2\right)$. We follow exactly the same procedure as in the proof of Lemma 16, except that $E_{14}^{\prime}=E_{14} / 16\left(1+y^{2}\right)^{2}$. It follows that the leading monomial of $R(x)$ is

$$
2^{64} d^{32} h^{16}\left(l^{2}+\left(h^{\prime}-d\right)^{2}\right)^{4}\left(l^{2}+\left(h^{\prime}+d\right)^{2}\right)^{4} x^{176} .
$$

Since $d$ and $h$ are not equal to $0, R(x) \equiv 0$ only if $l^{\prime}=0$ and $h^{\prime}= \pm d$. We replace $l^{\prime}$ by 0 and $h^{\prime}$ by $\pm d$ in $Q$ and $T$, and compute the new leading monomial of $R(x)$. In both cases, it is equal to

$$
2^{80} d^{32} h^{16} x^{160} .
$$

Therefore $R(x) \not \equiv 0$ and there is a finite number of distinct values of $\alpha=2 \arctan x$ in the set of solutions of System (20) in the variables $\left(\alpha, \alpha^{\prime}, \varphi, \varphi^{\prime}\right) \in\left(S^{1}\right)^{4}$.

The second equation of System (20) gives with the variable substitution $y=$ $\tan \left(\alpha^{\prime} / 2\right)$ that

$$
\frac{1-y^{2}}{1+y^{2}} \cos \alpha+\frac{2 y}{1+y^{2}} \sin \alpha+\frac{1-y^{2}}{1+y^{2}}=d
$$

or equivalently

$$
(d+1+\cos \alpha) y^{2}-2 \sin \alpha y+d-1-\cos \alpha=0 .
$$


This polynomial is not identically equal to zero since $d \neq 0$. Thus $\alpha$ determines at most two $\alpha^{\prime}=2 \arctan y$. It follows that there is a finite number of distinct values of $\alpha$ and $\alpha^{\prime}$ in the set of solutions of System (20) in the variables $\left(\alpha, \alpha^{\prime}, \varphi, \varphi^{\prime}\right) \in\left(S^{1}\right)$ t

Lemma 20. A couple of values $\left(\alpha, \alpha^{\prime}\right)$ determines $O(1)$ paths $\mathcal{P}$.

Proof. By Eqs. (21) and (23), $\alpha$ and $\alpha^{\prime}$ define uniquely $\theta$ and $x^{\prime}$ which characterize the position of $\mathcal{C}$ and $\mathcal{C}^{\prime}$. Path $\mathcal{P}$ is then characterized by the orientation of its four circular arcs.

\section{The algorithm}

Let $\mathcal{O}_{1}, \ldots, \mathcal{O}_{m}$ be the disjoint moderate obstacles. We denote by $\mathcal{S}_{O}$ the set of the obstacle edges and by $n$ its size.

Let $S$ and $F$ be the initial and final points of the optimal path we want to compute. By Theorems 3 and 4 , any $C$-segment is either an anchored $C$-segment, or is adjacent to a terminal $C$-segment and to an anchored $C$-segment, or belongs to a subpath of type $X S \bar{C} \bar{C} S X^{\prime}$ where $X, X^{\prime} \in\{O, \overline{\bar{C}}\}$ (the lengths of the $S$-segments being possibly zero).

The algorithm computes first the set $\mathcal{S}_{\overline{\bar{C}}}$ of all the maximal free anchored arcs of circle. A maximal free anchored arc is a maximal arc of an anchored circle that does not intersect the obstacles. It will be simply called a free anchored arc in the sequel.

For any given $r$, we associate to each obstacle a grown obstacle which is the Minkowski sum of the obstacle and of a disk of radius $r$. Let $\mathcal{A}_{r}$ be the arrangement of the boundaries of these grown obstacles. A point is said of level $i$ in $\mathcal{A}_{r}$ if it belongs to the interior of $i$ grown obstacles. The vertices of level 0 are simply the vertices of the boundary of the union of the grown obstacles. Because the obstacles are disjoint, there are $O(n)$ such vertices by a result of Kedem et al. ${ }^{16}$ The same bound holds for the number of vertices of the $k$ first levels for any constant $k$ by the random sampling theorem of Clarkson and Shor. ${ }^{10}$

Lemma 21. The number of free anchored arcs is $O(n)$ and these arcs can be computed in $O(n \log n)$ time.

Proof. Any moderate obstacle contains at least one disk of unit radius. Furthermore, at most five pairwise disjoint disks of unit radius can intersect any given circle of unit radius. Thus at most five pairwise disjoint moderate obstacles can intersect any given circle of unit radius. Hence any point lies in at most five obstacles grown by a disk of radius 1 . In other words, any point is of level at most five in $\mathcal{A}_{1}$. Therefore, $\mathcal{A}_{1}$ has linear size and can be computed in $O(n \log n)$ time by standard techniques. Finally, since the centers of the anchored circles are the vertices of $\mathcal{A}_{1}$ and each anchored circle is intersected by at most five obstacles, the lemma is proved. 
Once the free anchored arcs have been computed, we compute the set $\mathcal{S}_{S}$ of all the free line segments that are tangent to two arcs of $\mathcal{S}_{\overline{\bar{C}}} \cup \mathcal{S}_{O}$.

Lemma 22. $\mathcal{S}_{S}$ has size $O\left(n^{2}\right)$ and can be computed in $O\left(n^{2} \log n\right)$ time.

Proof. First, we compute all the free line segments tangent to two obstacles. Let us consider each obstacle in turn, say $\mathcal{O}_{1}$ for concreteness. Let $D_{\theta}$ be an oriented line of orientation $\theta$ tangent to $\mathcal{O}_{1}$. The set of common points between $\mathcal{O}_{1}$ and $D_{\theta}$ is either a single point or a line segment; let $P_{\theta}$ be either this single point or the middle point of the line segment. If $D_{\theta}$ intersects $\mathcal{O}_{i}(i=2, \ldots, m)$, we call $P_{i}$ the point common to $D_{\theta}$ and $\mathcal{O}_{i}$ that is closer to $P_{\theta}$. Let $f_{i}(\theta)$ be the algebraic length of $P_{\theta} P_{i}$ on the oriented line $D_{\theta}$.

Let $\mathcal{E}^{+}$(resp. $\mathcal{E}^{-}$) be the lower (upper) envelope of the functions $f_{i}$ that are positive (negative). As the obstacles are pairwise disjoint, $f_{i}(\theta) \neq 0$ and $f_{i}(\theta) \neq$ $f_{j}(\theta)$ for all $\theta$ and $i \neq j$. It follows that $\mathcal{E}^{+}$and $\mathcal{E}^{-}$can be computed in $O(n \log n)$ time. A line segment joining $P_{\theta}$ to $P_{i}$ is free if and only if $\left(\theta, f_{i}(\theta)\right)$ belongs to $\mathcal{E}^{+}$or $\mathcal{E}^{-}$. Moreover, a line segment $P_{\theta} P_{i}$ is tangent to $\mathcal{O}_{i}$ if and only if $P_{i}$ is an end-point of $f_{i}$. Hence, computing the free line segments tangent to $\mathcal{O}_{1}$ and another obstacle reduces to compute $\mathcal{E}^{+}$and $\mathcal{E}^{-}$.

Repeating the above procedure for all the obstacles, we conclude that all the free line segments tangent to two obstacles can be computed in $O\left(n^{2} \log n\right)$ time.

We now compute the free line segments tangent to a free anchored arc and to either an obstacle or another anchored free arc. Let $C_{1}, \ldots, C_{p}$ be the anchored free arcs. We consider in turn each free anchored arc, say $C_{1}$ for concreteness, and apply exactly the same procedure as above to compute the free line segments tangent to $C_{1}$ and to an obstacle. As above, these segments can be computed in $O(n \log n)$ time by computing the envelopes $\mathcal{E}^{+}$and $\mathcal{E}^{-}$. It remains to compute the free line segments tangent to $C_{1}$ and to the other anchored free $\operatorname{arcs} C_{2}, \ldots, C_{p}$. We define a function $g_{i}$ involving $C_{1}$ and $C_{i}$ and similar to the function $f_{i}$ defined above. To each end point of $g_{i}$ that lies between $\mathcal{E}^{+}$and $\mathcal{E}^{-}$corresponds a free line segment tangent to $C_{1}$ and $C_{i}$. Deciding if such an end point lies between $\mathcal{E}^{+}$and $\mathcal{E}^{-}$can be done in $O(\log n)$ time by binary search once the envelopes have been computed. As the number of free anchored $\operatorname{arcs}$ is $O(n)$ by Lemma 21, the free line segments tangent to $C_{1}$ and to another anchored free arc can be computed in $O(n \log n)$ time. Hence, the free line segments tangent to an anchored free arc and to either an obstacle or another anchored free arc can be computed in $O\left(n^{2} \log n\right)$ time in total.

This achieves the proof.

We consider now the subpaths of type $C_{t} C \overline{\bar{C}}$ and $\overline{\bar{C}} C C_{t}$. We compute the set $\mathcal{S}_{C}$ of all the circular arcs that avoid the obstacles and are tangent to a terminal circle and to an anchored free arc. As there are $O(n)$ anchored free arcs, this step can easily be done in $O\left(n^{2}\right)$ time. 
By Theorem 3, $\mathcal{S}_{\overline{\bar{C}}} \cup \mathcal{S}_{O} \cup \mathcal{S}_{S} \cup \mathcal{S}_{C}$ contains all the arcs potentially taken by an optimal path except the subpaths of type $X S \bar{C} \bar{C} S X^{\prime}$. We consider, in turn, all the quadruplets $\left(\mathcal{X}, \mathcal{O}, \mathcal{O}^{\prime}, \mathcal{X}^{\prime}\right)$ where $\mathcal{X}$ and $\mathcal{X}^{\prime}$ are obstacle edges or free anchored arcs, and where $\mathcal{O}$ and $\mathcal{O}^{\prime}$ are two obstacle edges. First, we compute a family of candidate subpaths of type $X S \bar{C} \bar{C}^{\prime} S X^{\prime}$ where $X$ (resp. $X^{\prime}$ ) is an arc of $\mathcal{X}\left(\mathcal{X}^{\prime}\right)$ and the two $C$-segments $\bar{C}$ and $\bar{C}^{\prime}$ are tangent to $\mathcal{O}$ and $\mathcal{O}^{\prime}$, respectively. In a second step, we check whether or not these potential optimal subpaths intersect other obstacles.

By Theorem 5, for any given choice of $\left(\mathcal{X}, \mathcal{O}, \mathcal{O}^{\prime}, \mathcal{X}^{\prime}\right)$, we can compute in $O(1)$ time a family of $O(1)$ candidate paths such that any optimal path of type $X S \bar{C} \bar{C}^{\prime} S X^{\prime}$, satisfying that neither $\bar{C}$ nor $\bar{C}^{\prime}$ is anchored, is contained in one of the candidate paths. Hence the total time complexity of this step, for all choices of $\left(\mathcal{X}, \mathcal{O}, \mathcal{O}^{\prime}, \mathcal{X}^{\prime}\right)$, is $O\left(n^{4}\right)$. We also compute the paths of type $X S \bar{C} \bar{C} S X^{\prime}$ and $X S \bar{C} \overline{\bar{C}} S X^{\prime}$ (where $X$ and $X^{\prime}$ are maximal). As the number of anchored $C$-segments is $O(n)$, the total number of these paths is $O\left(n^{4}\right)$ and they can be easily computed in $O\left(n^{4}\right)$ time. It remains to compute the set $\mathcal{S}_{\bar{C} \bar{C}}$ of those paths that avoid the obstacles.

Lemma 23. $\mathcal{S}_{\bar{C} \bar{C}}$ can be computed in $O\left(n^{4} \log n\right)$ time.

Proof. We show that we can check in $O(\log n)$ time whether or not a given subpath of type $X S \bar{C} \bar{C} S X^{\prime}$ intersects the obstacles. We consider successively the case of an arc of circle and the case of a line segment.

As mentioned in the proof of Lemma 21, a circle of unit radius intersects at most five obstacles. We can identify the obstacles that intersect the circle supporting a given $\operatorname{arc} \bar{C}$ by locating in $O(\log n)$ time the center of this circle in the arrangement $\mathcal{A}_{1}$. It then remains to check if the $\operatorname{arc} \bar{C}$ (not the whole circle) actually intersects one of the obstacles. Each such test can be done in $O(\log n)$ time since each obstacle has $O(n)$ edges. ${ }^{11}$

We describe now how to check if a line segment $S$ of a subpath of type $X S \bar{C} \bar{C} S X^{\prime}$ intersects the obstacles. By Lemma 8, the length of $S$ is at most 4 . It follows that if $S$ intersects an obstacle, each of its end points are contained in the obstacle grown by a disk of radius 4 . As the obstacles are disjoint and moderate, a point can only be contained in $g<36$ such grown obstacles ${ }^{\mathrm{c}}$; hence, arrangement $\mathcal{A}_{4}$ has linear size and can be computed in $O(n \log n)$ time. We locate one endpoint of $S$ in $\mathcal{A}_{4}$ and find the at most $g$ obstacles that might intersect $S$. We consider in turn each of these obstacles and check if $S$ indeed intersects the obstacle. This can be done in $O(\log n)$ time. $^{11}$

By Theorems $3, \mathcal{S}_{\overline{\bar{C}}} \cup \mathcal{S}_{O} \cup \mathcal{S}_{S} \cup \mathcal{S}_{C} \cup \mathcal{S}_{\bar{C} \bar{C}}$ contains all the arcs potentially taken by an optimal path. 
Let $\mathcal{G}$ be the weighted graph whose nodes are the tangent points between two arcs of $\mathcal{S}_{\overline{\bar{C}}} \cup \mathcal{S}_{O} \cup \mathcal{S}_{S} \cup \mathcal{S}_{C} \cup \mathcal{S}_{\bar{C} \bar{C}}$ and whose edges are the arcs of $\mathcal{S}_{\overline{\bar{C}}} \cup \mathcal{S}_{O} \cup \mathcal{S}_{S} \cup$ $\mathcal{S}_{C} \cup \mathcal{S}_{\bar{C} \bar{C}}$. The final step of the algorithm consists in searching a shortest path in this graph.

Theorem 6. An optimal path amidst a set of disjoint moderate obstacles with $n$ edges in total can be computed in $O\left(n^{4} \log n\right)$ time.

\section{Improving the performance of the algorithm}

We now show that the time complexity of the algorithm can be reduced in most practical situations. This is a consequence of the fact that the subpaths of type $\bar{C} \bar{C}$ can only be encountered near the endpoints of the path. Proposition 2 is a consequence of a claim of Agarwal et al. ${ }^{1}$ We give here a complete proof.

Proposition 2. Let $\mathcal{P}$ be an optimal path consisting of four parts $\mathcal{P}_{1}, \mathcal{P}_{2}, \mathcal{P}_{3}$ and $\mathcal{P}_{4}$ in this order where $\mathcal{P}_{2}$ and $\mathcal{P}_{3}$ are $C$-segments. Let $\mathcal{C}_{2}$ and $\mathcal{C}_{3}$ be the circles supporting $\mathcal{P}_{2}$ and $\mathcal{P}_{3}$, and let $\mathrm{O}_{2}$ and $\mathrm{O}_{3}$ be their centers.

(1) If $\mathcal{C}_{3}$ is not obstructed by any obstacle or if $\mathcal{C}_{2}$ is obstructed by an obstacle, then for any $M$ on $\mathcal{P}_{1}$, the Euclidean distance between $M$ and $O_{3}$ is smaller than 3.

(2) If $\mathcal{C}_{3}$ is obstructed by an obstacle or if $\mathcal{C}_{2}$ is not obstructed by any obstacle, then for any $M$ on $\mathcal{P}_{4}$, the Euclidean distance between $M$ and $O_{2}$ is smaller than 3.

Proof. We only prove the first claim; the second one is symmetrical. We assume, without loss of generality, that $\mathcal{P}_{2}$ and $\mathcal{P}_{3}$ are oriented counterclockwise and clockwise respectively.

We recall some notations indroduced in Section 2: let $M$ be a point of $\mathcal{P}$ and let $C_{L}(M)$ (resp. $C_{R}(M)$ ) be the unit circle tangent to $\mathcal{P}$ at $M$ and lying on the left (resp. right) side of path $\mathcal{P}$ oriented from $S$ to $T$. $C_{L}(M)$ is oriented counterclockwise and $C_{R}(M)$ is oriented clockwise. An arc of one of these circles will be oriented accordingly.

We show that for any $M$ on $\mathcal{P}_{1}, C_{L}(M)$ intersects $\mathcal{C}_{3}$. It will immediately follow that the Euclidean distance between $M$ and $O_{3}$ is smaller than 3 .

Suppose for a contradiction that there exists $M$ on $\mathcal{P}_{1}$ such that $C_{L}(M)$ does not intersect $\mathcal{C}_{3}$. Let $M_{0}$ be the point of $\mathcal{P}_{1}$ such that for any point $M$ located after $M_{0}$ on the relative interior of $\mathcal{P}_{1}, C_{L}(M)$ properly intersects $\mathcal{C}_{3}$ (see Figure 20); $M_{0}$ exists because $\mathcal{P}_{2}$ is greater than $\pi$ by Lemma 3 . Let $M_{1}$ be the common point to $C_{L}\left(M_{0}\right)$ and $\mathcal{C}_{3}$, and $M_{2}$ be the common end point of $\mathcal{P}_{2}$ and $\mathcal{P}_{3}$.

We distinguish whether $M_{1} \in \mathcal{P}_{3}$ or not. In each case we show that the dashed path of Figure 20 shortens $\mathcal{P}$ and avoids all the moderate obstacles, which contradicts the fact that $\mathcal{P}$ is optimal.

Case 1: $M_{1} \in \mathcal{P}_{3}$.

We first show that the $\operatorname{arc}\left[M_{0} M_{1}\right]$ of $C_{L}\left(M_{0}\right)$ is not obstructed by any moderate obstacle. A disk of unit radius that intersects $\left[M_{0} M_{1}\right]$ without intersecting the 


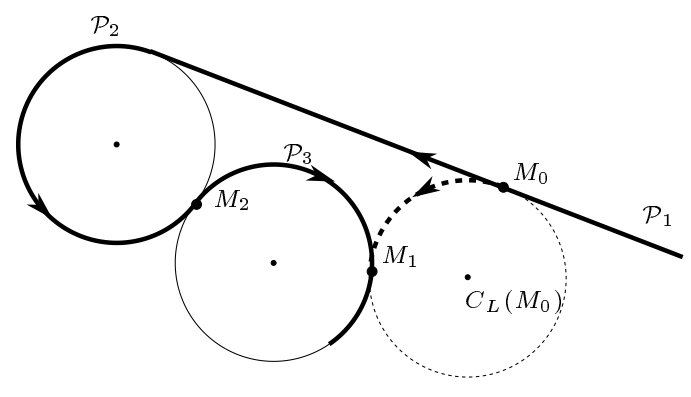

(a) $: M_{1} \in \mathcal{P}_{3}$

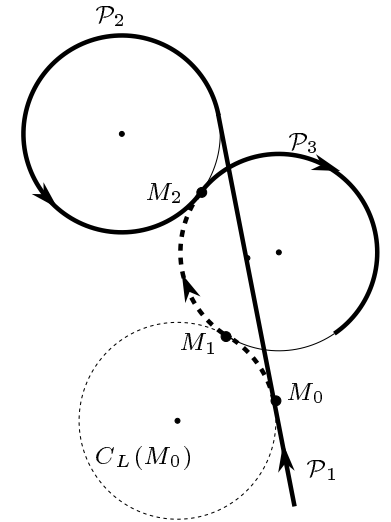

(b) : $M_{1} \notin \mathcal{P}_{3}$

Fig. 20. Shortcuts in the proof of Proposition 2.

portion $\mathcal{P}\left(M_{0}, M_{1}\right)$ of $\mathcal{P}$ between $M_{0}$ and $M_{1}$ is included in the region $\mathcal{R}$ inside the closed curve that is the concatenation of $\mathcal{P}\left(M_{0}, M_{1}\right)$ and of the $\operatorname{arc}\left[M_{1} M_{0}\right]$ of $C_{L}\left(M_{0}\right)$ (see Figure 21). By the definition of $M_{0}$, the relative interior of the arc of $\mathcal{P}_{1}$ located after $M_{0}$ is strictly included in the disk of radius 3 centered at $\mathrm{O}_{3}$. It follows that the only disks of unit radius included in $\mathcal{R}$ are the disks whose boundaries are $\mathcal{C}_{2}$ and $C_{L}\left(M_{0}\right)$ (which can possibly intersect). Since $\mathcal{P}_{2}$ is not an arc of the boundary of the obstacles by hypothesis, $\left[M_{0} M_{1}\right]$ is not obstructed by any moderate obstacle.

By the definition of $M_{0},\left[M_{0} M_{1}\right]$ is smaller than $\pi$ and so shortens $\mathcal{P}$ because $\mathcal{P}_{2}$ is strictly greater than $\pi$.

Case 2: $M_{1} \notin \mathcal{P}_{3}$.

First notice that the circle $\mathcal{C}_{2}$ is not obstructed by any obstacle. Indeed, otherwise $M_{0}$ must lie in the hashed region of Figure 22a and, as $\mathcal{P}_{3}$ is greater than $\pi$, $M_{1} \in \mathcal{P}_{3}$ which contradicts the hypothesis. By the hypothesis of the first claim of the proposition, it follows that $\mathcal{C}_{3}$ is not obstructed by any obstacle. That implies that the arc $\left[M_{0} M_{1}\right]$ of $C_{L}\left(M_{0}\right)$ avoids all the moderate obstacles because otherwise, the portion of $\mathcal{P}$ between $M_{0}$ and $M_{2}$ cannot lie entirely in the disk of radius 3 centered at $O_{3}$ (see Figure 22b), which contradicts the definition of $M_{0}$. Hence, the concatenation of the $\operatorname{arc}\left[M_{0} M_{1}\right]$ of $C_{L}\left(M_{0}\right)$ and the $\operatorname{arc}\left[M_{1} M_{2}\right]$ of $\mathcal{C}_{3}$ avoids all the moderate obstacles.

Moreover, the concatenation of $\left[M_{0} M_{1}\right]$ and $\left[M_{1} M_{2}\right]$ shortens $\mathcal{P}$. Indeed, the arc $\left[M_{0} M_{1}\right]$ is smaller than $\pi$ by the definition of $M_{0}$, and, the $\operatorname{arc}\left[M_{1} M_{2}\right]$ is smaller than $\pi$ because $M_{1} \notin \mathcal{P}_{3}$ and $\mathcal{P}_{3}$ is greater than $\pi$. It can be easily shown that the concatenation of two circular arcs of unit radius whose lengths are smaller than $\pi$ is a shortest path of bounded curvature (even if there is no obstacle). Hence, the concatenation of the $\operatorname{arcs}\left[M_{0} M_{1}\right]$ and $\left[M_{1} M_{2}\right]$ shortens $\mathcal{P}$ and avoids all the 


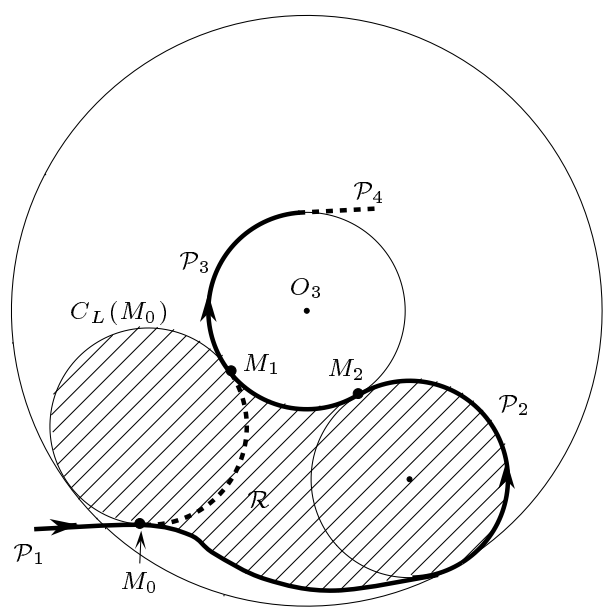

Fig. 21. For the proof of Proposition 2 (Case 1).

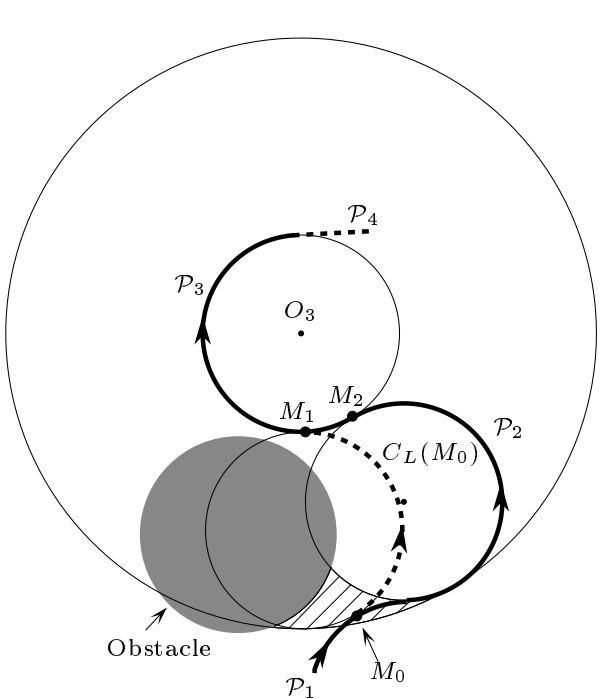

(a)

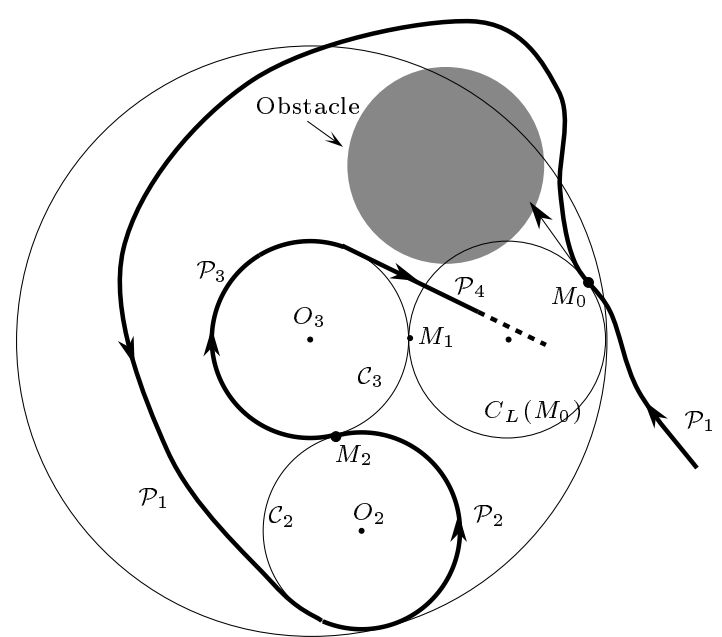

(b)

Fig. 22. For the contradictions in the proof of Proposition 2 (Case 2).

moderate obstacles which contradicts the hypothesis that $\mathcal{P}$ is optimal.

Remark 1. The previous proof yields that an optimal path contains at most two non-terminal $\bar{C} \bar{C}$-subpaths (i.e., subpaths of type $\bar{C} \bar{C}$ where both $\bar{C}$-segments are 


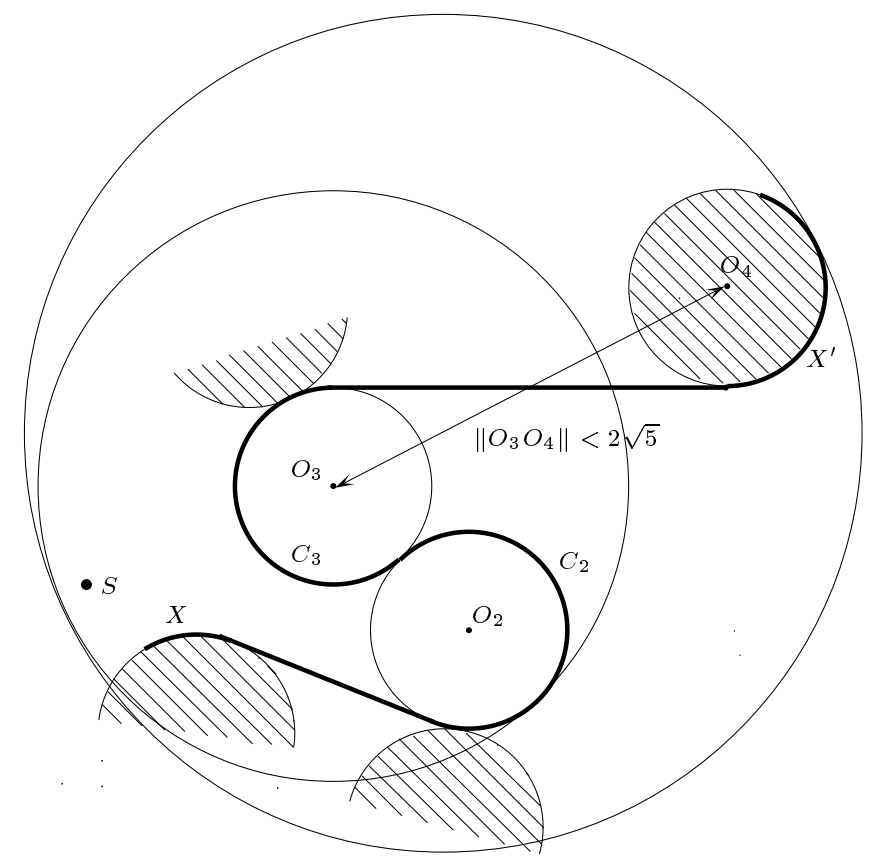

Fig. 23. For the proof of Theorem 7 .

not terminal). For details see Ref. 1.

Theorem 7. Let $\mathcal{P}$ be an optimal path joining $S$ to $F$. Any subpath of $\mathcal{P}$ of type $X S \bar{C} \bar{C} S X^{\prime}$, where $X, X^{\prime} \in\{O, \overline{\bar{C}}\}$, is contained in one of the two disks of radius 9 centered at $S$ or $F$.

Proof. This theorem follows from Proposition 2 and we use the notations introduced in that proposition. Let $\mathcal{P}_{1}$ (resp. $\mathcal{P}_{4}$ ) be the portion of $\mathcal{P}$ between $S$ (resp. $F)$ and the first (resp. last) $\bar{C}$ in the considered subpath. From Proposition 2, we have $\forall M \in \mathcal{P}_{1} M O_{3} \leq 3$ or $\forall M \in \mathcal{P}_{4} M O_{2} \leq 3$. Assume without loss of generality that $\forall M \in \mathcal{P}_{1} M O_{3} \leq 3$. Then, the starting point $S$ and the whole subpath $X S \bar{C} \bar{C}$ is included in the disk of radius 3 centered at $O_{3}$ (see Figure 23).

On the other hand, by Lemma 8 , the length of the line segment preceding $X^{\prime}$ is smaller than 4 . Therefore, $S$ and the whole subpath $X S \bar{C} \bar{C} S X^{\prime}$ is included in a disk of diameter $3+2 \sqrt{5}+1<9$ (see Figure 23). Hence the subpath of type $X S \bar{C} \bar{C} S X^{\prime}$ is included in a disk of radius 9 centered at $S$.

According to Theorem 7, we can improve the procedure that computes the subpaths of type $X S \bar{C} \bar{C} S X^{\prime}$. Indeed, instead of considering all $n^{4}$ quadruplets $\left(\mathcal{X}, \mathcal{O}, \mathcal{O}^{\prime}, \mathcal{X}^{\prime}\right)$, we can only consider those that intersect one of the disks of radius 
9 centered at $S$ and $F$. If $k$ is the number of such quadruplets, the time complexity of the algorithm becomes $O\left(n^{2} \log n+k^{4} \log k\right)$. In particular, if the length of any obstacle edge is bounded from below by some positive constant, then $k=O(1)$.

Theorem 8. Given a set of disjoint moderate obstacles with n edges whose lengths are bounded from below by some positive constant, an optimal path between two configurations amidst these obstacles can be computed in $O\left(n^{2} \log n\right)$ time.

\section{Conclusion}

In this paper, we have considered disjoint moderate obstacles whose boundaries consist of $n$ line segments and circular arcs of unit radius. We have presented an $O\left(n^{4} \log n\right)$ time algorithm for computing shortest paths of bounded curvature. Moreover, if the length of the obstacle edges is bounded from below by some positive constant, then the time complexity of our algorithm reduces to $O\left(n^{2} \log n\right)$.

The algorithm we have presented in this paper works even if the moderate obstacles are not disjoint. However, its time complexity increases since the number of anchored $C$-segments might then be quadratic. A simple analysis of our algorithm shows that its time complexity is then $O\left(n^{7}\right)$.

It would be interesting to consider more general moderate obstacles (in the sense of Agarwal et al.) and, in particular, obstacles whose boundaries consist of line segments and circular arcs of radii greater than or equal to 1 . When considering such obstacles, the necessary conditions (9) and (20) for a subpath to be optimal become slightly more complicated. Unfortunately, the computations of resultants, performed in the proofs of Lemmas 16 and 19, then exceed the capabilities of the current computer algebra systems ${ }^{\mathrm{d}}$.

Finally, note that if the obstacles are polygonal, one can compute their convex hull of bounded curvature (see Ref. 6) and then compute shortest paths of bounded curvature amidst these new obstacles.

Many other questions remain open. We mention two of them: Can similar results be obtained for polygonal robots? Can similar results be obtained if backwards moves are allowed? (Preliminary results in that direction can be found in Refs. 1, 5 and 21.)

\section{Acknowledgements}

The authors would like to thank the GDR MEDICIS (GDR CNRS 1026) for giving them the oportunity to use the machines of the GAGE group at Ecole Polytechnique.

\footnotetext{
${ }^{\mathrm{d}}$ Using AXIOM, the size of the process exceeds 500MB.
} 


\section{References}

1. P. K. Agarwal, P. Raghavan and H. Tamaki, Motion planning for a steering-constrained robot through moderate obstacles, in Proc. 27th Annual ACM Symp. on Theory of Computing, ACM, New York (1995) pp. 343-352.

2. J. Barraquand and J.-C. Latombe, Nonholonomic multi-body mobile robots: Controllability and motion planning in the presence of obstacles, Algorithmica 10 (1993) 121155.

3. H. Behnke, F. Bachmann, K. Fladt and W. Suss, Fundamentals of mathematics, Geometry, volume 1, MIT Press, 1986.

4. J.-D. Boissonnat and X.-N. Bui, Accessibility Region for a Car that Only Moves Forwards along Optimal Paths, Research Report 2181, INRIA, Le Chesnay Cedex, France, 1994.

5. J.-D. Boissonnat, A. Cérézo and J. Leblond, Shortest paths of bounded curvature in the plane, Internat. J. Intell. Syst. 10 (1994) 1-16.

6. J.-D. Boissonnat and S. Lazard, Convex hulls of bounded curvature, in Proc. 8th Canadian Conference on Computational Geometry, Ottawa (1996) pp. 14-19.

7. J.-D. Boissonnat and S. Lazard. A polynomial-time algorithm for computing a shortest path of bounded curvature amidst moderate obstacles. In Proc. 12th Annu. ACM Symp. on Computational Geometry, Philadelphia, PA (1996) pp. 242-251.

8. X.-N. Bui, P. Souères, J.-D. Boissonnat and J.-P. Laumond, The shortest path synthesis for non-holonomic robots moving forwards, in Proc. IEEE Internat. Conf. on Robotics and Automation, San Diego, CA (1994) pp. 2-7.

9. L. Cesari, Optimization, theory and applications, Springler-Verlag, 1983.

10. K. L. Clarkson and P. W. Shor, Applications of random sampling in computational geometry, II, Discrete Comput. Geom. 4 (1989) 387-421.

11. D. P. Dobkin and D. L. Souvaine, Computational geometry in a curved world, Algorithmica 5 (1990) 421-457.

12. L. E. Dubins, On curves of minimal length with a constraint on average curvature and with prescribed initial and terminal positions and tangents, Amer. J. Math. 79 (1957) 497-516.

13. S. Fortune and G. Wilfong, Planning constrained motion, Ann. Math. Artificial Intelligence 3 (1991) 21-82.

14. P. Jacobs, Minimal length curvature constrained paths in the presence of obstacles, Research Report 90042, LAAS, 7, av du colonel-Roche 31077 Toulouse Cedex, 1990.

15. P. Jacobs and J. Canny, Planning smooth paths for mobile robots, in Nonholonomic Motion Planning, eds. Z. Li and J. Canny (Kluwer Academic Publishers, Norwell, MA, 1992) 271-342.

16. K. Kedem, R. Livne, J. Pach, and Micha Sharir, On the union of Jordan regions and collision-free translational motion amidst polygonal obstacles, Discrete Comput. Geom. 1 (1986) 59-71.

17. J.-C. Latombe, Robot Motion Planning, Kluwer Academic Publishers, Norwell, MA, 1991.

18. S. Lazard, Planification de trajectoires de robots mobiles non-holonomes et de robots à pattes, Thèse de doctorat en sciences, université Paris 6, France (1996).

19. Z. Li and J.F. Canny, Nonholonomic Motion Planning, Kluwer Academic Publishers, 1992.

20. J.-P. Laumond, P. Jacobs, M. Taix and R. Murray, A motion planner for nonholonomic mobile robots, IEEE Trans. Robotics Automation, 10 (1994) 577-593.

21. J. A. Reeds and L. A. Shepp, Optimal paths for a car that goes both forwards and backwards, Pacific J. Math. 145 (1990) 367-393. 
22. H. J. Sussmann and G. Tang, Shortest Paths for the Reeds-Shepp Car: A Worked Out Example of the Use of Geometric Techniques in Nonlinear Optimal Control, Report SYCON-91-10, Rutgers University, New Brunswick, NJ, 1991.

23. H. Wang and P. K. Agarwal, Approximation algorithms for curvature-constrained shortest paths, in Proc. 7th ACM-SIAM Symp. on Discrete Algorithms, Atlanta, GA (1996) pp. 409-418. 